\title{
Non-universal gaugino mass models under the lamppost of muon (g-2)
}

\author{
Joydeep Chakrabortty, ${ }^{a}$ Arghya Choudhury $^{b}$ and Subhadeep Mondal ${ }^{b}$ \\ ${ }^{a}$ Department of Physics, Indian Institute of Technology, \\ Kanpur-208016, India \\ ${ }^{b}$ Regional Centre for Accelerator-based Particle Physics, \\ Harish-Chandra Research Institute, Allahabad 211019, India \\ E-mail: joydeep@iitk.ac.in, arghyachoudhury@hri.res.in, \\ subhadeepmondal@hri.res.in
}

ABSTRACT: In unified $\mathcal{N}=1$ supergravity scenario the gaugino masses can be nonuniversal. The patterns of these non-universalities are dictated by the vacuum expectation values of non-singlet chiral super-fields in visible sector. Here, we have analysed the model independent correlations among the gaugino masses with an aim to explain the $[1 \div 3] \sigma$ excess of muon (g-2) $\left(\Delta a_{\mu}\right)$. We have also encapsulated the interconnections among other low and high scale parameters, compatible with the collider constraints, Higgs mass, relic density and flavour data. We have noted that the existing non-universal models are not capable enough to explain $\Delta a_{\mu}$ at $[1 \div 2] \sigma$ level. In the process, we have also shown the impact of recent limits from the searches for disappearing track and long lived charged particles at the LHC. These are the most stringent limits so far ruling out a large parameter space allowed by other constraints. We have also performed model guided analysis where gaugino masses are linear combination of contributions coming from singlet and non-singlet chiral super-fields. Here, a new mixing parameter has been introduced. Following the earlier methodology, we have been able to constrain this mixing parameter and pin down the promising models on this notion.

KeYwords: Supersymmetry Phenomenology

ARXIV EPRINT: 1503.08703 


\section{Contents}

1 Introduction 1

2 Generation of gaugino masses in $\mathcal{N}=1$ unified SUGRA models 3

3 Muon g-2 5

4 Collider, low energy and dark matter searches constraints $\quad 7$

$\begin{array}{ll}4.1 \text { Collider constraints } & 7\end{array}$

4.2 Flavour physics data 11

$\begin{array}{lll}4.3 & \text { Dark matter constraints } & 11\end{array}$

5 Strategy of analysis $\quad 12$

6 High scale non-universality vs muon g-2 12

6.1 Non-universal gauginos and universal scalars - model independent analysis 12

$\begin{array}{lll}6.1 .1 & \text { Relic density } & 15\end{array}$

$\begin{array}{lll}\text { 6.1.2 Direct and indirect detections } & 17\end{array}$

$\begin{array}{ll}\text { 6.1.3 Some benchmark points } & 18\end{array}$

6.2 Universal gauginos and non-universal scalars - model independent analysis 18

6.3 Non-universal gauginos and universal scalars - model based analysis 20

7 Conclusion $\quad 22$

\section{Introduction}

Supersymmetry (SUSY) is one of the most promising beyond Standard Model (BSM) scenarios that solves the gauge hierarchy problem, stabilizes the Higgs mass and also addresses some of the other shortcomings of the SM. It also provides a weakly interacting massive particle (WIMP) which can be a viable cold dark matter (DM). In R-parity conserving scenario, the lightest supersymmetric particle (LSP) happens to be that DM candidate. From unification view point, SUSY shows an improvement over the Standard Model (SM) predictions with a consistent grand unification (GUT) scale.

In global minimal supersymmetric standard model (MSSM), the gauge group is same as that for the SM, but the particle content is extended in form of the supersymmetric partners. In unbroken SUSY scenario, the supertrace ${ }^{1}$ vanishes exactly which is due to the degeneracy in the spectrum and equality in fermionic and bosonic degrees of freedoms in theory. However, so far experimental data suggests that all the SUSY particles must be heavier than their respective SM partners. Thus the supertrace must be non-vanishing and, in general, proportional to the SUSY scale. In other words, we can say that SUSY can be

\footnotetext{
${ }^{1}$ Supertrace in supersymmetric theory is defined as: $\mathcal{S} t r=\sum_{s}(-1)^{2 s}(2 s+1) \operatorname{Tr}\left(m_{s}^{2}\right)$, where $s, m_{s}$ are the spin and mass of the particle respectively.
} 
realized in nature only in broken form. ${ }^{2}$ So, the important question that rises in this context is following: what is the SUSY breaking mechanism? So far we have failed to pin down any specific breaking scenario, rather fenced by different possibilities. These mechanisms can be broadly classified into two categories: spontaneous ${ }^{3}$ and explicit breaking by addition of the SUSY breaking soft terms in the Lagrangian [3-9]. The later one is not forbidden by any physical principle but it leads to enormous number of free parameters (more than 100) in the theory and spoils the beauty of it. We are not elaborating this possibility as this is not the prime moto of this paper. The outcome of spontaneous breaking depends on which field is getting vacuum expectation value (VEV). However, as we know, spontaneous breaking does not change the property of the action. Hence the supertrace which is a signature of the Lagrangian, is kept unmodified. This is against the experimental observation as mentioned before and thus ruled out. Again if we start with an exact SUSY Lagrangian, we are bound to break SUSY only spontaneously [3,4]. This dilemma can be resolved if one breaks SUSY in the hidden sector. This information of SUSY breaking can be brought to the visible sector by different messengers, giving rise to different models, such as gravity-, gauge-, anomaly-mediated SUSY scenarios. For the sake of our work, let us further proceed our discussion on the gravity mediated SUSY breaking scenario: $\mathcal{N}=1$ SUGRA [10-21]. In the minimal SUGRA (mSUGRA) framework, there are only 5 free parameters compared to over 100 in the general MSSM case: universal scalar mass $\left(m_{0}\right)$, universal gaugino mass $\left(m_{1 / 2}\right), \tan \beta$ (ratio of the up and down type Higgs VEVs $=v_{u} / v_{d}$ ), tri-linear coupling $\left(A_{0}\right)$ and sign of $\mu$ term $(\operatorname{sgn}(\mu))$. However, it is not necessary for the gauginos to be degenerate at the high scale itself. If the visible sector possesses an unified gauge symmetry, the gauginos may become non-degenerate through the VEV of the GUT breaking scalars [22-34]. Then the high scale input parameters consist of $m_{0}, M_{3}, \tan \beta, A_{0}$ and $\operatorname{sgn}(\mu)$, where, $M_{3}$ is the mass scale for the $\mathrm{SU}(3)_{C}$ gauginos. This also determines $M_{1}$ and $M_{2}$, as they are correlated: $M_{1}: M_{2}: M_{3}=a: b: c$, where $a, b, c$ depend on patterns of the symmetry breaking. This enriches the possibility of having different correlations among these gaugino masses which lead to different compositions of the LSP at low scale. Thus unlike the mSUGRA case, here, the LSP can be bino, wino, higgsino or admixture of these three states. This allows us to explore wide range of phenomenologies driven by non-universal gaugino masses. The phenomenological aspects of non-universal gaugino mass scenario are discussed in [35-64]. Due to its varieties of LSP configurations, these models have significant impact on the analyses regarding DM searches and muon (g2) excess. These two issues have been explored extensively within mSUGRA framework. But the present limits on squarks and gluino put severe stringent bounds on mSUGRA parameter space. This results in unavailability of SUSY spectrum within this framework that can explain $\Delta a_{\mu}{ }^{4}$ at $[1 \div 2] \sigma$ level respecting flavour constraints.

\footnotetext{
${ }^{2}$ In passing we would like to draw attention to another view as suggested [1] questioning the necessity of supersymmetry breaking.

${ }^{3}$ Dynamical supersymmetry breaking is an interesting possibility [2] but is troublesome as it might lead to charge and colour breaking vacua.

${ }^{4} \Delta a_{\mu}$ denotes the discrepancy between experimentally measured value of muon (g-2) and the SM predicted one.
} 
This has been the motivation of our work. The prime aim of our analysis is to understand the model independent correlations among the MSSM gauginos which can successfully explain the anomalous muon (g-2) excess over the SM, and simultaneously satisfy different experimental bounds at low energy. In this context we have discussed two scenarios: non-universal gaugino \& universal scalars, universal gaugino \& non-universal scalars. We have extended our former framework by adopting existing SUSY-GUT models and considering the general gaugino spectrum. We also introduce here a new mixing parameter that is related to the superpotentials. We have encapsulated the range of this new parameter while explaining muon $(\mathrm{g}-2)$ excess at $[1 \div 3] \sigma$ level.

\section{Generation of gaugino masses in $\mathcal{N}=1$ unified SUGRA models}

Here, we have briefly reviewed the Lagrangian based discussion on the generation of gaugino masses. In $\mathcal{N}=1$ supergravity framework, the part of the needful Lagrangian which is associated with the gauge and gaugino sectors is expressed as [13-16, 24-30]

$$
\begin{aligned}
\mathbf{e}^{-1} \mathcal{L}= & -\frac{\mathcal{F}_{\alpha \beta}}{2} \frac{\overline{\lambda^{\alpha}} \not D \lambda^{\beta}}{2}-\frac{1}{4} \operatorname{Tr}\left[F_{\mu \nu} \mathcal{F}(\Phi) F^{\mu \nu}\right] \\
& +\frac{1}{4} e^{-G / 2} G^{i}\left(G^{-1}\right)_{i}^{j}\left[\frac{\delta \mathcal{F}_{\alpha \beta}^{*}\left(\Phi^{*}\right)}{\delta \Phi^{j *}}\right] \lambda^{\alpha} \lambda^{\beta}+\text { h.c. },
\end{aligned}
$$

where, $\alpha, \beta, \gamma=[1, \ldots, A d j] .{ }^{5}$ Here, we have set $M_{P l} / \sqrt{8 \pi}=1$. Our nomenclature is following: $G, \mathcal{F}, \Phi$ and $\lambda$ are the Kähler potential, the super-potential, chiral super-fields and gaugino fields respectively.

Here, the derivatives of Kähler potential are defined as: $G^{i}=\frac{\delta G}{\delta \Phi_{i}}, G_{j}^{i}=\frac{\delta^{2} G}{\delta \Phi^{* j} \delta \Phi_{i}}$, and $\left(G^{-1}\right)_{j}^{i}$ is the inverse of $G_{j}^{i}$. The chiral function $\mathcal{F}_{\alpha \beta}(\Phi)$ is an analytic function of $\Phi$. These $\Phi$ 's are the chiral super-fields belonging to the visible $(\phi)$ as well as the hidden $\left(z_{h}\right)$ sectors. The chiral field $z_{h}$ is singlet under the gauge symmetry of the visible sector. The other chiral super-field $\phi$ is non-singlet under such gauge symmetries. The choices of $\phi$ are further restricted from the gauge kinetic term $\operatorname{Tr}\left[F_{\mu \nu} \mathcal{F}(\Phi) F^{\mu \nu}\right]$ which dictates that $\phi$ can only belong to the symmetric product of two adjoint representations. In this framework, both SUSY and unified gauge symmetry are broken spontaneously. The VEV of $z_{h}\left(z_{h}^{0}\right)$ that breaks local SUSY spontaneously is very large $\left(\sim 10^{19} \mathrm{GeV}\right)$ where the VEV of $\phi$ $\left(V_{\mathrm{GUT}}\right)$ sets the GUT scale $\left(M_{U}\right)$. The generic structure of the chiral function, $\mathcal{F}_{\alpha \beta}$, can be given as suggested in $[28,30]$ (neglecting higher order terms which are more suppressed):

$$
\mathcal{F}_{\alpha \beta}=\mathcal{F}_{1} \delta_{\alpha \beta}+\mathcal{F}_{2} c_{\alpha \beta \gamma} \phi^{\gamma}
$$

where $\mathcal{F}_{1,2}$ are the functions of chiral super-fields of hidden and visible sectors, and $c_{\alpha \beta \gamma}$ are the group theoretic factors. The first term is the canonical term leading to mSUGRA like scenario where all the gauginos are degenerate, i.e., universal. The second term is due to the presence of GUT symmetry breaking scalars which cause splitting in universal gaugino spectrum of mSUGRA leading to non-universal gaugino masses at the high scale

\footnotetext{
${ }^{5}$ For $\mathrm{SU}(N)$ and $\mathrm{SO}(N), \operatorname{Adj}$ is defined as $\left(N^{2}-1\right)$ and $\left(N^{2}-N\right) / 2$ respectively.
} 

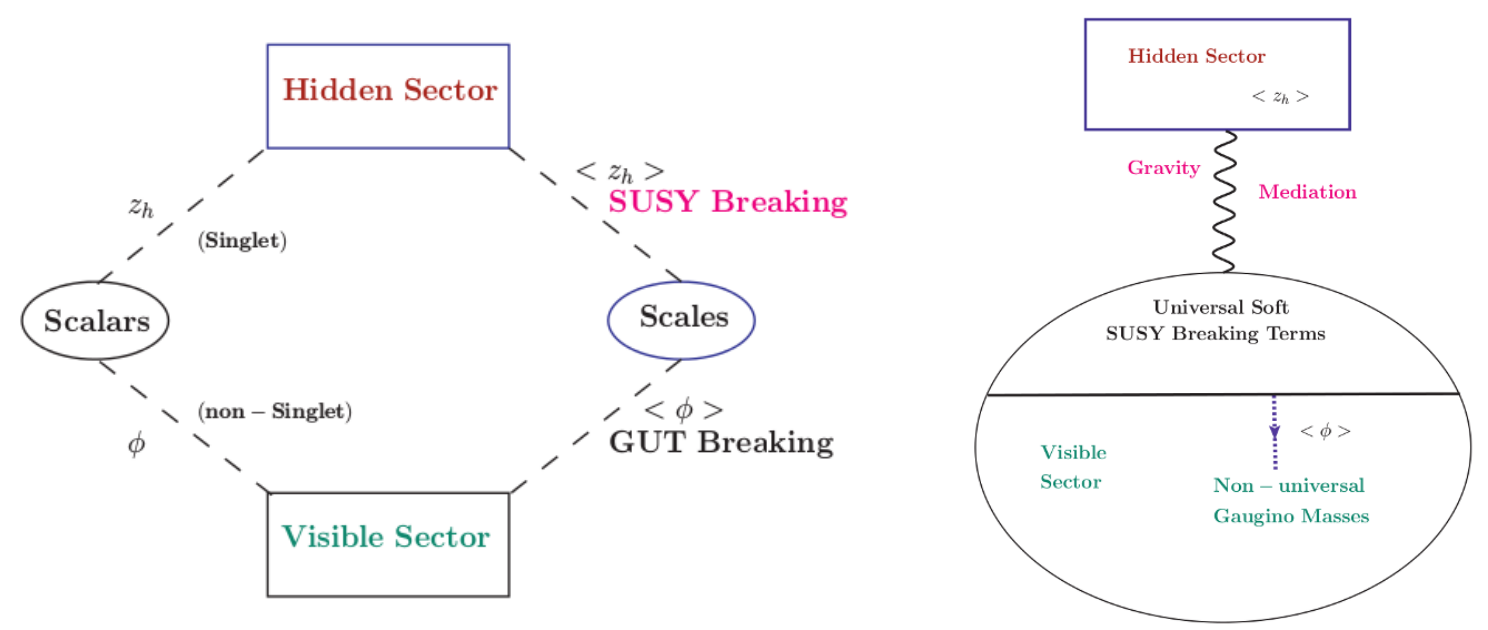

Figure 1. Schematic flow chart to demonstrate spontaneous breaking of supersymmetry in the hidden sector through the VEV of the singlet chiral super-field $\left(z_{h}\right)$ and successive breaking of GUT symmetry in the visible sector through the VEV of non-singlet chiral super-filed $(\phi)$. As the outcome of this process, the soft SUSY breaking terms are generated in the visible sector and the gauginos become non-degenerate at the high scale itself.

itself. The different choices of these $\phi$ fields lead to possible non-universalities as the high scale boundary conditions. For the purpose of our present analysis, we consider that $\mathcal{F}_{1,2}$ are the only functions of $z_{h}$, and $\phi$. In figure 1 , we have summarized this whole process through a schematic flow chart.

Once SUSY and GUT are broken, one can recast the gaugino mass terms as

$$
M_{i}=M\left[P+\delta_{i} V_{\mathrm{GUT}} Q\right],
$$

where the $\delta_{i}$ 's are the group theoretical factors for broken and unbroken generators (see refs. [28-30] for details) similar to $c_{\alpha \beta \gamma}$ in eq. (2.2). We can write down $i \ni[j, X]$ where $j$ and $X$ represent the indices for unbroken and broken generators respectively. To understand this better, let us consider the breaking of $\mathrm{SU}(5)$ to $\mathrm{U}(1)_{Y} \otimes \mathrm{SU}(2)_{L} \otimes \mathrm{SU}(3)_{C}$ $\left(\mathcal{G}_{M S S M}\right)$. As an outcome of this mechanism, 12 generators of $\mathcal{G}_{M S S M}$ are unbroken, and rest of the $(24-12)=12$ generators are broken. In our further analysis, we denote the gauginos corresponding to the broken generators as $X$ and others are associated with index $j$. Here, $P$ and $Q$ are the functions of $z_{h}^{0}, V_{\mathrm{GUT}}$ and derivatives of $\mathcal{F}_{1,2}$, evaluated at $\left[z_{h}^{0}\right.$, $\left.V_{\mathrm{GUT}}\right]$. Within SUGRA framework, unfortunately, the exact functional forms of $\mathcal{F}_{1}, \mathcal{F}_{2}$ are unknown and same for $P, Q$. However, in simplified scenario, like mSUGRA, due to absence of GUT group in the visible sector there is no need of non-singlet field $\phi$ in the theory. This implies that all the $\delta_{i}$ 's are identically zero leading to all the gaugino masses to be degenerate, i.e., universal.

But within the unified SUGRA framework, the generic form of the gaugino mass is given in eq. (2.3) which cannot be further simplified to understand the specific correlations among them unless we make some assumption. In refs. [31-34] it was pointed out from pure phenomenological perspective that if $P / Q$ is negligibly small then the ratio of the gaugino 
masses are just the ratios of $\delta_{i}$ 's. It has been also argued in refs. $[28,30]$ that to stabilise the cosmological constant, the masses of the $X$-gauginos must vanish and also provide unique mass ratios for MSSM gauginos independent of $P$ and $Q$. Here, the gaugino masses are automatically non-universal and posses definite non-universal patterns. To complete this discussion, we would like to mention that the generation of gaugino masses in context of supergravity framework was first discussed in refs. [28-30] for SU(5) unified theory. Later this has been generalised for $\mathrm{SO}(10)$ and $E(6)$ GUT groups in refs. [31-34].

The generic form of gaugino masses, without any further assumption, can be recast as:

$$
\mathcal{M}_{i}=M^{\prime}\left[1+\wp \delta_{i}\right]
$$

where $\wp$ is the ratio of $P, Q$ and can be thought of as a measure of mixing between singlet and non-singlet contributions. We will emphasize more on this mixing parameter, $\wp$, from muon (g-2) point of view for some specific models.

\section{Muon g-2}

The precision measurements are leaving very little room for new physics. One of them which has been measured experimentally with an immaculate accuracy is the muon anomalous magnetic moment. In the $\mathrm{SM}$, there exists a tree level contribution to $\operatorname{muon}\left(\ell_{\mu}\right)$-muonphoton $\left(A_{\rho}\right)$ coupling $\left(i e \overline{\ell_{\mu}} \gamma^{\rho} \ell_{\mu} A_{\rho}\right)$. Along with that in BSM scenario, the relatively heavier and so far unobserved particles may contribute to this vertex through radiative corrections in the form of an effective operator like $\left(i e / 4 m_{\mu}\right) a_{\mu} \overline{\ell_{\mu}}\left[\gamma^{\lambda}, \gamma^{\rho}\right] \ell_{\mu}\left(\partial_{\lambda} A_{\rho}-\partial_{\rho} A_{\lambda}\right)$. So far, there is a discrepancy, $\Delta a_{\mu}$, among the SM theoretical prediction and experimentally observed value $[65,66]$. Thus the BSM contributions, if there is any, have to be fitted within the deviation $[67]$

$$
\Delta a_{\mu}=(29.3 \pm 9.0) \times 10^{-10}
$$

It is expected that SUSY can explain this excess through the exchanges of different sparticles and that has been one of the motivation for TeV scale SUSY. The loop induced contributions (see figures 2, 3) involving sleptons, neutralinos, charginos and sneutrinos are very important in this regard [68-71]. In the context of general SUSY scenario, muon (g-2) has been discussed in [72-79]. Within the unified SUGRA framework the contributions to anomalous muon magnetic moment are discussed in [79-86].

In this paper, we have started with a framework where all the scalar masses are universal but the gauginos are non-degenerate at the high scale itself. The other parameters, say, $\tan \beta, \operatorname{sgn}(\mu), A_{0}\left(=-2 m_{0}\right), m_{H_{u}}^{2}=m_{H_{d}}^{2}\left(=m_{0}^{2}\right)$ are chosen suitably. Our intention is to adjudge the patterns of non-universalities in gaugino masses under the light of $\Delta a_{\mu}$ at $[1 \div 3] \sigma$ level. In other words, we have used $\Delta a_{\mu}$ to intrigue unified SUGRA model building.

Computation of the radiative contributions to $a_{\mu}$ is very similar to the generic SUSY scenario. The only difference is in the generation of the low scale SUSY spectrum. Due to the high scale boundary conditions, our scenario is much more constrained. Thus the low scale sparticle spectrum cannot be tuned arbitrarily to fulfil the necessary contributions for muon (g-2). In general the chargino-sneutrino loop (see figure 3) contributes more 

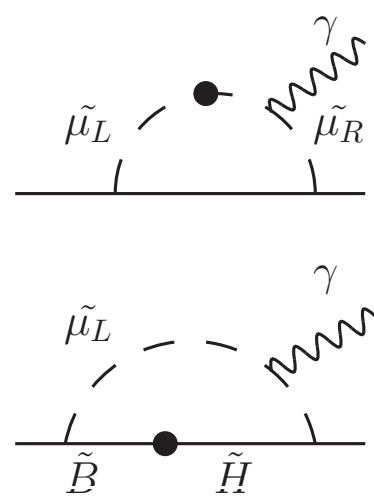
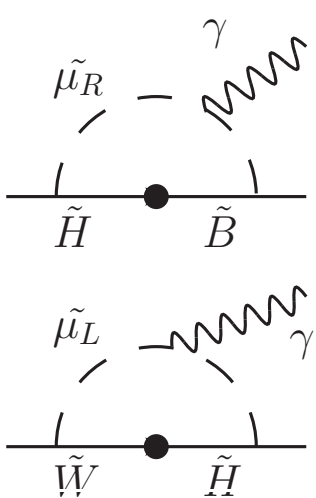

Figure 2. Feynman diagrams that contribute to muon (g-2) involving neutralinos $(\tilde{B}, \tilde{W}, \tilde{H})$ and smuons $\left(\tilde{\mu}_{L, R}\right)$.

dominantly compared to the neutralino-smuon loop (see figure 2). But this is not always true as these contributions depend on the right-handed smuon $\left(\tilde{\mu}_{R}\right)$ mass.

The mas parameters masses of sleptons, neutralinos, charginos and sneutrinos play crucial roles to determine the muon anomalous magnetic moment. The high scale parameters which are involved in these computations are $M_{1,2}, m_{0}\left(\equiv \tilde{\mu}_{L, R}\right), \tan \beta, A_{0}$ and $\operatorname{sgn}(\mu)$. The dependence of $\Delta a_{\mu}$ on $\tan \beta$ is linear which is due to the requirement of chirality flipping through the Yukawa couplings. Thus $\Delta a_{\mu}$ is very sensitive to $\tan \beta$ and larger value of $\tan \beta$ is favoured to produce bigger contribution to $\Delta a_{\mu}$. However, we are forced to respect the constraints from flavour data and need to be careful while considering large $\tan \beta$. We would like to notify that the choice of $\operatorname{sgn}(\mu)$ is also restricted as $\mu M_{3}<0$ is severely constrained from the measurement of $\operatorname{BR}(b \rightarrow s \gamma)$. Also note that $\Delta a_{\mu}$ prefers $\operatorname{sgn}(\mu)>0$. Hence throughout this work, we only consider $\mu>0$.

In our analysis, the scalar masses are universal at the high scale, thus the splitting between left- and right-handed sleptons are not large. However, through the renormalisation group evolutions, the off-diagonal terms in the slepton mass matrix can be generated and that may lead to an open possibility of having contributions from all generations of sleptons. Again if the $\mu$ term, i.e., the higgsino mass parameter is larger than the masses of the left-handed smuons then the masses of the right-handed smuons play crucial role (see left-top of figure 2). Then the contribution to $\Delta a_{\mu}$ from this diagram decreases as mass of the right-handed smuon increases. Note that this effect is only visible if the diagrams in figure 2 dominate over the one in figure 3. In general, if the lightest neutralino, lighter chargino, left- \& right-handed smuons and sneutrinos are nearly degenerate (within few tens of $\mathrm{GeV}$ ) and also $\mu$ term is of the same order, then the following diagrams dominantly contribute positively to $a_{\mu}$ (see top-left of figure 2 and figure 3 ). Note that if the sneutrinos are lighter than the smuons and also the $\mu$ term (as in our case), the dominant contribution may come from figure 3 . 


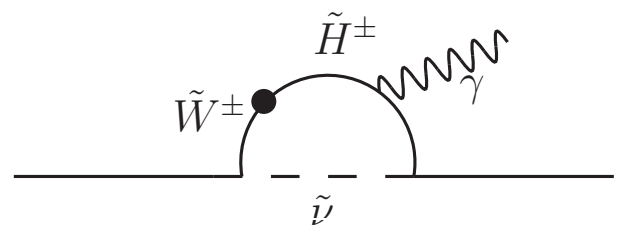

Figure 3. Feynman diagrams that contribute to muon (g-2) involving charginos $\left(\tilde{W}^{ \pm}, \tilde{H}^{ \pm}\right)$and sneutrino $(\tilde{\nu})$.

\section{Collider, low energy and dark matter searches constraints}

The recent searches at LHC continue to put severe exclusion limits on sparticle masses and couplings. In case of SUSY, the parameter space is very sensitive to the mass of the observed Higgs boson and other flavour data. Along with these collider constraints one cannot ignore the impact of DM searches (direct, indirect) and also needs to respect the upper bound of relic density. Here, we catalogue the imposed constraints in our analysis.

\subsection{Collider constraints}

In this section, we first discuss the limits obtained from LEP (I \& II) and then from the LHC searches. The latest search results for long-lived charged particles and disappearing tracks at the LHC are also crucial for our study. Thus we prefer to discuss these separately at the end of this subsection.

\section{LEP exclusions}

We have implemented the following LEP constraints on the masses of sparticles:

- sleptons: $m_{\widetilde{l}_{L}}, m_{\widetilde{l}_{R}}>100.0 \mathrm{GeV}$ where $l=e, \mu ; m_{\widetilde{\tau}_{1}}>86.6 \mathrm{GeV}$,

- lighter chargino: $m_{\tilde{\chi}_{1}^{ \pm}}>103.5 \mathrm{GeV}$.

More details on these exclusions are discussed in ref. [87].

\section{LHC exclusions}

After the completion of LHC Run-I, the exclusion limits on the sparticle masses are much more stronger now. More specifically, due to the large production cross-sections, constraints on the masses of strongly interacting SUSY particles (e.g., gluino, squarks) are more restrictive. Both ATLAS and CMS collaborations have updated their analyses in $n$ leptons $+m$-jets ( with or without $b$-tagging) $+\mathbb{F}_{T}$ (where $n$ and $m$ can take values $0,1,2, \ldots$ ) channels for supersymmetric models like mSUGRA, cMSSM and other simplified SUSY scenarios. Apart from the production cross-section, all the limits also crucially depend on the branching ratios (BRs) and mass separations among the mother and daughter sparticles.

In SUGRA type scenarios, all the gaugino $\left(\widetilde{\chi}_{1}^{0}, \widetilde{\chi}_{1}^{ \pm}, \widetilde{g}\right)$ masses are correlated: either universal or non-universal at the GUT scale itself. As we are focussing on non-universal gaug- 
ino scenario, we have incorporated the limits coming from the searches of simplified models with suitable changes ${ }^{6}$ for the sake of our analysis rather using direct mSUGRA bounds.

We have mentioned earlier that at the electro-weak (EW) scale, the contributions to muon (g-2) are controlled by the following parameters: masses of sleptons, lighter chargino, lightest neutralino and their compositions. As we are working within an unified framework at the high scale, the low scale spectrum is correlated with the high scale soft SUSY breaking terms and the hierarchy in low scale spectrum is determined by other parameters too. For example, one can accommodate light sleptons even satisfying present limits on heavy gluino $\left(m_{\tilde{g}}\right)$ and squarks $\left(m_{\tilde{q}}\right)$ masses.

Higgs mass. We allow only those high scale parameters that lead to the lightest Higgs boson mass to be: $M_{h}=(122 \div 128) \mathrm{GeV}$ considering a theoretical uncertainty of $\sim$ $3 \mathrm{GeV}[88,89]$.

Limits on chargino and neutralino masses. Most of the LHC analyses on direct chargino searches $[90,91]$ are based on the assumption that the LSP is bino type and the lighter chargino is wino type. For higgsino or mixed type LSP and(or) chargino, the production cross-sections of $\widetilde{\chi}_{1}^{ \pm} \widetilde{\chi}_{2}^{0}$ reduce significantly and the limits from trilepton data $[90,91]$ become much weaker. Again the limits are slightly sensitive to the masses of sleptons ${ }^{7}$ where the LHC collaborations have assumed the slepton masses to be in the midway between $m_{\widetilde{\chi}_{1}^{ \pm}}$and $m_{\widetilde{\chi}_{2}^{0}}$. For bino type LSP and wino type lighter chargino, we have implemented the conservative limits in our analysis. Here, we have supplemented the mass limits mentioning specific hierarchical scenarios:

- If $m_{\widetilde{l}}<m_{\widetilde{\chi}_{1}^{ \pm}}, m_{\widetilde{\chi}_{2}^{0}}$ : for $m_{\widetilde{\chi}_{1}^{0}}<300 \mathrm{GeV}, m_{\widetilde{\chi}_{1}^{ \pm}}$is excluded below $720 \mathrm{GeV}[90,91]$. With an conservative approach having $m_{\widetilde{\chi}_{1}^{0}}$ in between 300 to $400 \mathrm{GeV}, m_{\widetilde{\chi}_{1}^{ \pm}}$is excluded in the range of 400 to $700 \mathrm{GeV}$ and for $m_{\widetilde{\chi}_{1}^{0}}>400 \mathrm{GeV}$, the limits are not applicable [90, 91].

- For heavy slepton scenarios $\left(m_{\widetilde{l}}>m_{\widetilde{\chi}_{1}^{ \pm}}, m_{\widetilde{\chi}_{2}^{0}}\right): \widetilde{\chi}_{1}^{ \pm}$and $\widetilde{\chi}_{2}^{0}$ decay via $W$ and $Z$ bosons and the limits on chargino masses are relatively weaker. Again the lighter chargino mass below $420 \mathrm{GeV}$ is excluded with $m_{\widetilde{\chi}_{1}^{0}} \lesssim 140 \mathrm{GeV}$ [90].

- When the $\widetilde{\chi}_{2}^{0} \rightarrow h \widetilde{\chi}_{1}^{0}$ decay mode dominates, the limits become much weaker in the heavy slepton scenarios. Masses of $\widetilde{\chi}_{1}^{ \pm}$and $\widetilde{\chi}_{2}^{0}$ are excluded upto $240 \mathrm{GeV}$ for a massless $\widetilde{\chi}_{1}^{0}$ and the limits are invalidated for $m_{\widetilde{\chi}_{1}^{0}}>40 \mathrm{GeV}[93]$.

\footnotetext{
${ }^{6}$ The results obtained in simplified models are mainly based on the assumption of single particle pair production and one or two step decay modes. Thus one needs to be very careful while using these bounds. The ideal situation would be to calculate the production cross-section for each and every SUSY spectrum and compute the the LHC bounds accordingly. But this is beyond the scope of our analysis. The methodology that has been adapted in this paper is conservative and more importantly not capable enough to alter our predictions.

${ }^{7}$ For varying slepton masses and different mass hierarchies, using the LHC $8 \mathrm{TeV}$ data the limits are revisited in a recent analysis [79]. For revised mass limits in higgsino dominated and(or) mixed chargino scenarios see [92].
} 
- With light third generation slepton $\left(m_{\widetilde{\tau}_{1}}<m_{\widetilde{\chi}_{1}^{ \pm}}, m_{\widetilde{\chi}_{2}^{0}}\right): m_{\widetilde{\chi}_{1}^{ \pm}}$upto $380 \mathrm{GeV}$ is excluded for $m_{\widetilde{\chi}_{1}^{0}}<85 \mathrm{GeV}$ [90].

Limits on slepton masses. ATLAS and CMS have searched for charged sleptons (first two generations) from direct production of $\mathrm{R}$ (ight) and(or) $\mathrm{L}$ (eft)-type sleptons [91, 94]. ATLAS limits are slightly stronger than that suggested by CMS. Though we have implemented the limits for R-, L- and $\mathrm{R}=\mathrm{L}$-types separately, here, we have discussed only that for $\mathrm{R}=\mathrm{L}$-type slepton scenario as suggested by ATLAS collaboration using di-lepton search channel [94]. The masses of selectron and smuon (both L- and R-type) are excluded in the mass region between $[90 \div 325] \mathrm{GeV}$ with massless $m_{\widetilde{\chi}_{1}^{0}}$. But the sensitivity of exclusion limit is relaxed once the slepton-LSP mass splitting decreases. For example, for $m_{\widetilde{\chi}_{1}^{0}}=$ $100 \mathrm{GeV}$, common left- and right-handed slepton masses within $[160 \div 310] \mathrm{GeV}$ are excluded. We have adopted the conservative bin wise limits on slepton masses from figure 8 of ref. [94]. For example, slepton mass is excluded within $[268 \div 310] \mathrm{GeV}$ for $m_{\widetilde{\chi}_{1}^{0}}[170 \div 180]$ $\mathrm{GeV}$. We have to keep in mind that these limits exist for LSP mass upto $180 \mathrm{GeV}$.

Limits on gluino mass. At the LHC, squarks and gluino pair production cross-sections are the largest among other SUSY production channels. The limits on gluino mass $\left(m_{\tilde{g}}\right)$ crucially depend on the decay properties of $\widetilde{g}$ and(or) mass hierarchy between squarks, gluino and other sparticles. If the squarks are lighter than the gluino $\left(m_{\widetilde{g}}>m_{\widetilde{q}}\right)$, gluino decays via $\widetilde{q} q$ and for such scenario when all squarks and gluinos are produced, the limits on the strong sector of sparticle is most stringent. In mSUGRA (cMSSM) type of scenarios with $\tan \beta=30, A_{0}=-2 m_{0}$ and $\mu>0$, degenerate squarks and gluinos are excluded for masses upto $1.7 \mathrm{TeV}$ at $95 \% \mathrm{CL}$ [95]. This limit is applicable for relatively small universal scalar mass $m_{0}$ (typically $<1 \mathrm{TeV}$ ). For large $m_{0}$ and small $m_{1 / 2}$ (say, $\sim[500 \div 600] \mathrm{GeV}$ ), gluino decays into $\widetilde{t}_{1} t$ (note that, the first two generation squarks are much heavier than stop). In such scenarios (with top quark dominated final states), gluino masses smaller than $1.4 \mathrm{TeV}$ are excluded from $[0-1] \ell+3$ b jets $+\mathbb{F}_{T}$ analysis [96].

In simplified scenarios where gluino decays as: $\widetilde{g} \rightarrow \widetilde{q} q \rightarrow q \bar{q} \widetilde{\chi}_{1}^{0}$ ( $q$ denotes first two generation squarks) then $m_{\widetilde{g}}$ upto 1.5 to $1.55 \mathrm{TeV}$ is excluded for $m_{\widetilde{\chi}_{1}^{0}}$ upto $600 \mathrm{GeV}$ [95]. When the squarks are very heavy and gluino decays via $\widetilde{g} \rightarrow q \bar{q} \widetilde{\chi}_{1}^{0}$ (first two generations) then $m_{\widetilde{g}}$ below $1.4 \mathrm{TeV}$ is excluded for $m_{\widetilde{\chi}_{1}^{0}} \lesssim 300 \mathrm{GeV}$ [95]. All these limits are weakened considerably for the compressed scenarios. ${ }^{8}$ For example, when the difference between $m_{\widetilde{g}}$ and $m_{\widetilde{\chi}_{1}^{0}}$ is very small then the exclusion limits on $m_{\widetilde{g}}$ reduces to $\sim[550 \div 600] \mathrm{GeV}$ (for details see figure 10a of ref. [95]). Even for other scenarios or decay patterns, gluino mass below 1.1 to $1.3 \mathrm{TeV}$ is excluded for relatively light neutralino. The detail reports on SUSY searches regarding various limits for different scenarios, one can consult refs. [102, 103].

Limits on squarks mass. As discussed earlier, squark masses are excluded upto $1.7 \mathrm{TeV}$ when they are degenerate with gluino. For very heavy gluino scenarios we have implemented the following constraints in our analysis:

\footnotetext{
${ }^{8}$ In compressed scenarios, due to small mass difference between the mother and daughter particle, the missing energy $\left(\mathbb{F}_{T}\right)$ and transverse momentum $\left(p_{T}\right)$ of the jets or leptons become softer. Eventually the limits become weaker [97-101].
} 
- Light flavoured squark masses are excluded below $850 \mathrm{GeV}$ for $m_{\widetilde{\chi}_{1}^{0}} \leq 350 \mathrm{GeV}$ [95].

- For the decay modes $\widetilde{t}_{1} \rightarrow t \widetilde{\chi}_{1}^{0}$, the exclusion limit on $m_{\widetilde{t}_{1}}$ is upto $[600 \div 700] \mathrm{GeV}$ for $m_{\widetilde{\chi}_{1}^{0}} \leq 250 \mathrm{GeV}[104,105]$. Again when stop decays as $\widetilde{t}_{1} \rightarrow b \widetilde{\chi}_{1}^{ \pm}$, depending on the assumption over the chargino masses, ATLAS and CMS collaborations have excluded stop mass upto $[500 \div 600] \mathrm{GeV}$ for $m_{\widetilde{\chi}_{1}^{0}}$ upto $[200 \div 250] \mathrm{GeV}[104,106]$. For other decay modes, like $\widetilde{t}_{1} \rightarrow c \widetilde{\chi}_{1}^{0} ; b W \widetilde{\chi}_{1}^{0} ; b f f^{\prime} \widetilde{\chi}_{1}^{0}$, these limits become weaker: $m_{\widetilde{t}_{1}} \geq[240 \div 260] \mathrm{GeV}[104,106,107]$.

- Mass of sbottom below $620 \mathrm{GeV}$ is excluded at 95\% CL when LSP mass is $\leq 150 \mathrm{GeV}$ [108]. For small mass difference between sbottom and LSP, exclusion limit is pushed upto $250 \mathrm{GeV}$ [107].

\section{LHC searches on heavy charged particles}

\section{Limits from the search for disappearing track}

ATLAS and CMS have recently presented searches for charginos based on the high- $p_{T}$ disappearing tracks ${ }^{9}$ when the $\widetilde{\chi}_{1}^{ \pm}$is nearly degenerate with $\widetilde{\chi}_{1}^{0}$. For $\Delta M=m_{\widetilde{\chi}_{1}^{ \pm}}-m_{\widetilde{\chi}_{1}^{0}}$ $=140(160) \mathrm{MeV}$, ATLAS and CMS have excluded $m_{\tilde{\chi}_{1}^{ \pm}}$upto $500(270) \mathrm{GeV}$, see figure 5 in [110] (CMS) and figure 7 in [111] (ATLAS) for details. We incorporate these exclusion contours obtained by ATLAS and CMS to find the compatible parameter space in our analysis. We have to keep in mind that this limit is applicable only when both the lightest neutralino and lighter chargino are wino like.

\section{Limits from the search for long lived charged particle}

ATLAS collaboration has published the exclusion limits which are out come of searches for heavy long-lived charged particles using $8 \mathrm{TeV}$ data ${ }^{10}[112]$. When the charginos $\left(\widetilde{\chi}_{1}^{ \pm}\right)$ are nearly degenerate with $\widetilde{\chi}_{1}^{0}$, i.e., $\Delta M<135 \mathrm{MeV}$, then the exclusion limit on chargino mass becomes more stringent: $m_{\widetilde{\chi}_{1}^{ \pm}}>620 \mathrm{GeV}$ [112]. CMS has also presented a similar analysis $[113,114]$ and the results are in well agreement with ATLAS.

We have noted that these two above mentioned searches play crucial roles and lead to most stringent constraints. We have discussed the impact of these new limits in the following sections. In passing we would like to mention that both these exclusion limits, for nearly degenerate scenarios, crucially depend on the composition of chargino. For higgsino dominated lighter chargino these limits are relaxed considerably.

\footnotetext{
${ }^{9}$ In the nearly degenerate scenarios, $\widetilde{\chi}_{1}^{ \pm}$decays via $\pi^{ \pm} \widetilde{\chi}_{1}^{0}$ [109]. Due to the small mass difference between $\widetilde{\chi}_{1}^{ \pm}$and $\widetilde{\chi}_{1}^{0}$, the phase space is limited and the chargino has a significant lifetime. On the other hand, the daughter pion has momentum of $\sim 100 \mathrm{MeV}$ which is typically too small for its track to be reconstructed. For charginos that decay inside the tracker volume resulting in a disappearing track.

${ }^{10}$ When charged particles travel with speed slower than the speed of light, they can be identified and their mass can be determined from their measured speed and momentum. ATLAS collaboration has measured these quantities using time of flight and specific ionisation energy loss.
} 


\subsection{Flavour physics data}

- The measured value of $\mathrm{BR}(b \rightarrow s \gamma)$ does agree moderately well with the SM prediction and leaves very little room to fit BSM contribution within it. Thus this BR turns out to be a severe constraint whose impact can not be unnoticed. In the MSSM framework, the charged Higgs and chargino exchange diagrams may contribute dominantly to this branching ratio. Since light chargino boosts the required enhancement in $\Delta a_{\mu}$, this constraint is very important for our study. However, the contributing diagrams to $\operatorname{BR}(b \rightarrow s \gamma)$ interfere destructively if $\mu$ and $A_{t}$ are of opposite signs. Hence we choose to work with a positive $\mu$ and negative $A_{t}$ throughout. We impose the following constraint for all our points: $2.77 \times 10^{-4}<\operatorname{BR}(b \rightarrow s \gamma)<4.09 \times 10^{-4}$ (at $3 \sigma$ level) [115].

- The flavour physics constraint coming from the measurement of $\operatorname{BR}\left(B_{s} \rightarrow \mu^{+} \mu^{-}\right)$ puts strong bounds on the MSSM parameter space. For large $\tan \beta$, this branching ratio is proportional to $(\tan \beta)^{6}$ and inversely proportional to $m_{A}^{4}$. Thus this is expected to be a critical constraint for large $\tan \beta$ scenarios which are favoured to boost the enhancement in $\Delta a_{\mu}$. We impose $0.67 \times 10^{-9}<\operatorname{BR}\left(B_{s} \rightarrow \mu^{+} \mu^{-}\right)<$ $6.22 \times 10^{-9}$ (at $2 \sigma$ level) $[116,117]$ as a constraint throughout our study.

\subsection{Dark matter constraints}

\section{Relic density}

In this work, we have combined WMAP nine year data [118] (2 $\sigma$ bound) with $10 \%$ error in theoretical estimation which together propel the upper bound of relic density to 0.138 . Thus including this range, the $3 \sigma$ limit as suggested by the PLANCK [119] can be written as:

$$
0.092<\Omega h^{2}<0.138
$$

Here, we have adopted the eCMB $+\mathrm{BAO}+\mathrm{H}_{\mathrm{O}}$ combined value of table 4 in ref. [118].

In our case, lightest neutralino $\widetilde{\chi}_{1}^{0}$, i.e., the LSP, is the dark matter candidate. Instead of taking the $2 \sigma$ window, we have respected only the upper limit of $\Omega h^{2}$. This is because the DM candidate need not to be necessarily single-component but can also be a multi-component one [120-128]. Although while presenting our benchmark points, we only consider those points which produce the perfect relic density, i.e, $0.1145<\Omega h^{2}<0.1253$.

\section{Direct detection}

Apart from the relic density upper limit, we also discuss the implication of direct detection of DM using XENON100 [129] and LUX [130] data on spin independent neutralino-proton $\widetilde{\chi}_{1}^{0} p$ scattering cross-section $\left(\sigma_{\widetilde{\chi}_{1}^{0} p}^{S I}\right)$. The $t$-channel Higgs and $s$-channel squark exchange diagrams contribute to $\sigma_{\widetilde{\chi}_{1}^{0}}^{S I}$. For heavy squark scenario, the dominant contribution to the cross-section comes from the Higgs exchange diagram [131]. Again if $\widetilde{\chi}_{1}^{0}$ has sufficiently large higgsino component then $\sigma_{\widetilde{\chi}_{1}^{0} p}^{S I}$ may become large [132]. 


\section{$5 \quad$ Strategy of analysis}

The prime aim of our analysis is to understand the correlations of the MSSM gaugino masses at the high scale itself which can explain the $[1 \div 3] \sigma$ excess of muon (g-2). In the process, we have imposed several constraints, namely, bounds from collider searches and Higgs mass. We have also forced our solutions to respect flavour constraints and the upper bound of relic density. The recent searches for disappearing tracks and long lived charged particles by ATLAS and CMS have put very stringent constraints on the parameter space. In our analysis, particularly, these have played very crucial roles.

To find the model independent correlations among $M_{1}, M_{2}$ and $M_{3}$ we have treated these gaugino masses as individual free parameters and varied over wide ranges along with other free parameters, like $m_{0}$ and $\tan \beta$. The tri-linear coupling $A_{0}$ is taken to be $-2 m_{0}$ and Higgs parameters are set as $m_{H_{u}}^{2}=m_{H_{d}}^{2}=m_{0}^{2}$. Using these high scale input parameters, we have generated the SUSY spectrum at low scale and sorted out the parameter space which is compatible with the above mentioned constraints. We have noted the interconnections among different set of high and low scale parameters which have been discussed in detail in later section. The same strategy has been implemented to identify the mixing among singlet and non-singlet contributions for different SUSY-GUT models from muon (g-2) window.

We have generated SUSY spectrum using SuSpect (v2.41) [133]. Further, for DM relic density, direct-indirect detection cross-section and flavour physics calculation we have used micrOMEGAs (v3.6.7) [134] and calcHEP (v3.3.6) [135]. In our analysis, muon (g-2), has been computed using micrOMEGAs.

\section{High scale non-universality vs muon g-2}

\subsection{Non-universal gauginos and universal scalars - model independent analysis}

For the sake of our analysis we have supplemented the following input parameters at the high scale: $m_{0}, M_{1}, M_{2}, M_{3}, \tan \beta, \operatorname{sgn}(\mu)$. All the gaugino mass parameters $\left(M_{1}, M_{2}, M_{3}\right)$ are varied randomly and individually at the high scale over a wide range along with other parameters. Below we have listed the ranges of parameters that we have considered for our detailed analysis:

$$
\begin{aligned}
& m_{0} \in(1,3000) \mathrm{GeV} \text {; } \\
& A_{0}=-2 m_{0} ; \\
& M_{1} \in(200,5000) \mathrm{GeV} \text {; } \\
& M_{2} \in(-5000,+5000) \mathrm{GeV} \text {; } \\
& M_{3} \in(-5000,+5000) \mathrm{GeV} \text {; } \\
& \tan \beta \in(1,60) \text {. }
\end{aligned}
$$

We have mentioned earlier that the most stringent constraint is appearing for nearly degenerate lighter chargino $\left(m_{\widetilde{\chi}_{1}^{ \pm}}\right)$\& lightest neutralino $\left(m_{\widetilde{\chi}_{1}^{0}}\right)$ scenario. We have noted that for a large part of the parameter space that leads to $[1 \div 2] \sigma$ excess in $\Delta a_{\mu}$ contain nearly degenerate $\widetilde{\chi}_{1}^{ \pm}$and $\widetilde{\chi}_{1}^{0}$. Understandably, the $1 \sigma$ points appear only at the low mass region for $\widetilde{\chi}_{1}^{0}$ and hence also for $\widetilde{\chi}_{1}^{ \pm}$. In this type of scenario, a consequence of having a 


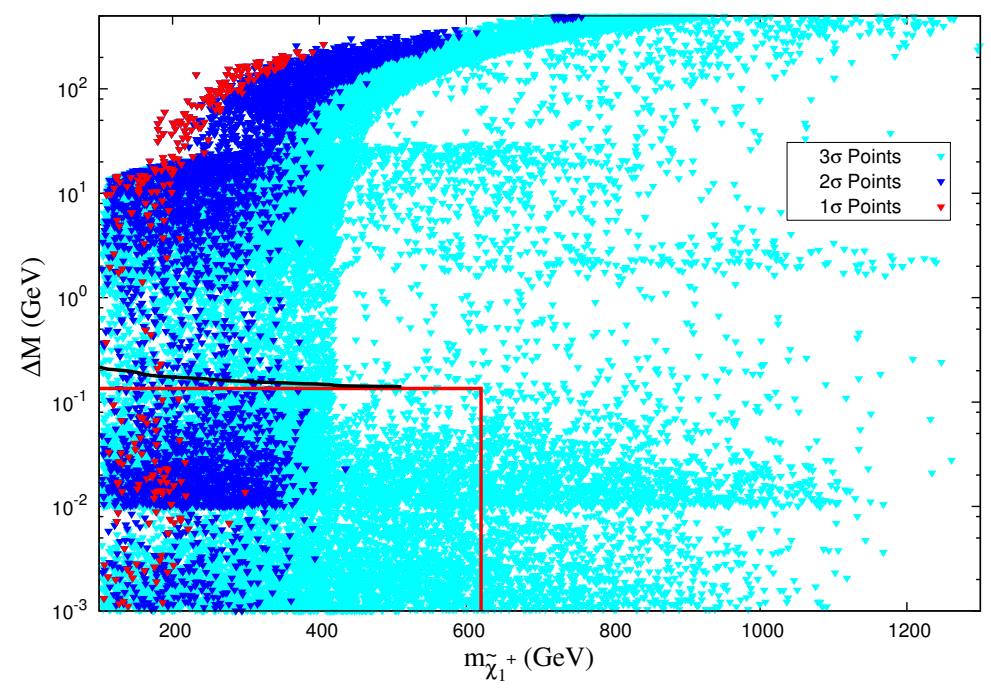

Figure 4. Impact of constraints from search for disappearing track and long lived particles, see section 4.1. The region enclosed by the red lines is excluded from long-lived or stable charged particle search. The area under the slanted black line just above the horizontal red line is excluded from disappearing charged track search at the LHC.

wino-like LSP is that the lighter chargino can then be as light as the LSP, provided one has much heavier higgsinos what is exactly what we have here. If $\widetilde{\chi}_{1}^{ \pm}$mass is just above the $\widetilde{\chi}_{1}^{0}$ mass so that it can decay into a charged pion $\left(\pi^{ \pm}\right)$and the LSP, one observes a charge track at the detector. This puts a strong limit on the chargino mass [112]. Now if the degeneracy between the $\widetilde{\chi}_{1}^{ \pm}$and $\widetilde{\chi}_{1}^{0}$ masses is such that $\widetilde{\chi}_{1}^{ \pm}$cannot decay further, its mass limit becomes even stronger [110, 111]. Figure 4 shows the distribution of the measure of mass degeneracy $\Delta M$, as a function of chargino mass. The red, blue and cyan colours represent the points that signify $1 \sigma, 2 \sigma$ and $3 \sigma$ excess in $\Delta a_{\mu}$ respectively. The exclusion limit $[110,111]$ from long-lived or stable $m_{\widetilde{\chi}_{1}^{ \pm}}$is shown by the red rectangle at the left bottom corner of the plot. As one can see, all the points below $\Delta M=135 \mathrm{MeV}$ are excluded for a chargino mass upto $620 \mathrm{GeV}$. The region under the slanted black line just above the horizontal red line is excluded from disappearing track search of charged particle [112]. This, by far, appears to be the strongest constraint ${ }^{11}$ for a wino-like LSP scenario.

From this point onwards, we consider only those points which survive the constraints imposed by the charged track and stable charged particle search besides all the other collider, DM and flavour constraints as described in section 4. Colour coding corresponding to the $1 \sigma, 2 \sigma$ and $3 \sigma$ reaches of muon (g-2) remain unchanged in the rest of the paper. In figure 5 we have shown the distribution of the obtained gaugino mass ratios $\left(\frac{M_{i}}{M_{3}}, i=1,2\right)$. We have chosen the range of gaugino masses to encapsulate all the four possible correlations between the ratios $M_{13}\left(\frac{M_{1}}{M_{3}}\right)$ and $M_{23}\left(\frac{M_{2}}{M_{3}}\right)$ including their signs.

\footnotetext{
${ }^{11}$ In passing we would like to mention that the numbers we have quoted for the particle masses are obtained from SuSpect [133] which includes one-loop corrections [136]. However, if one incorporate the two-loop corrections, the maximum mass splitting can be reduced by 2 to $5 \mathrm{MeV}$ [137] depending upon the SUSY scale. Thus the real impact of this two loop correction will be hardly visible in our figure 4 .
} 


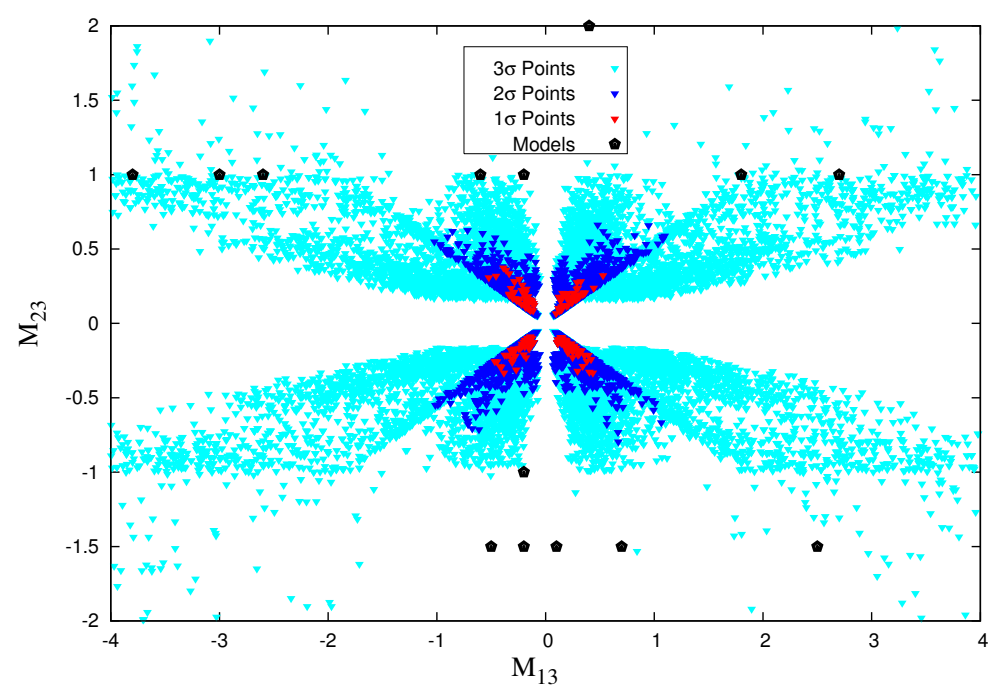

Figure 5. Model independent correlations among $M_{1}, M_{2}, M_{3}$ depending on $[1 \div 3] \sigma$ muon (g-2) excess after satisfying all constraints discussed in the text. The black dots represent the models encapsulated in tables 1, 2 in ref. [42]. Here, we have shown only few example model points where other models are out of this frame.

Figure 5 is almost symmetric and all the quadrants are in same footing for the purpose of our analysis. Hence it is sufficient to concentrate on one of the four quadrants to understand the nature of the distribution. It is clear that for an enhancement of $\Delta a_{\mu}$ at $[1 \div 2] \sigma$ level, one needs to have $\left|M_{23}\right| \leq 0.7$ and $\left|M_{13}\right| \leq 1.1$ excluding $M_{i 3}=0$ for $i=1,2$. From these ratios, one can have an idea about the composition of the LSP under consideration. The mass splitting increases as the contribution of bino component increases in the LSP state. As evident from figure 4 the mass splitting can be as large as $300 \mathrm{GeV}$ to produce the required $[1 \div 2] \sigma$ enhancement in $\Delta a_{\mu}$.

Note that, in this analysis, $\mu$ parameter is always relatively higher than $M_{1}$ and $M_{2}$ at the low scale. This a consequence of setting the Higgs soft masses $\left(m_{H_{d}}\right.$ and $\left.m_{H_{u}}\right)$ equal to $m_{0}$ at the high scale. Hence there is no possibility of having a small higgsino component that can contribute to the LSP state. Then it is evident that the most dominating contribution to the muon (g-2) enhancement would come from the processes involving light wino-like $\tilde{\chi}_{1}^{ \pm}$and light sleptons. This is one of the reason why the constraints from the search of heavy charged particles are so proactive in our case and rule out a large portion of the parameter space.

The other parameter that plays a very important role in the muon $(\mathrm{g}-2)$ calculation is $\tan \beta$. As discussed earlier, the SUSY contribution to muon (g-2) is directly proportional to $\tan \beta$. As a result, large $\tan \beta$, say $[8 \div 35]$, is favoured to achieve $1 \sigma$ enhancement which is evident from the figure 6 . However, the $2 \sigma$ enhancement can be obtained for a $\tan \beta$ as low as 5 and as high as 47 .

One can infer from the contributing diagrams in figure 2 that light slepton and LSP are crucial for the enhancement since they only appear as propagators. From figure 6, we observe that the LSP mass must be lighter than $400 \mathrm{GeV}$ for our purpose. Similarly, 

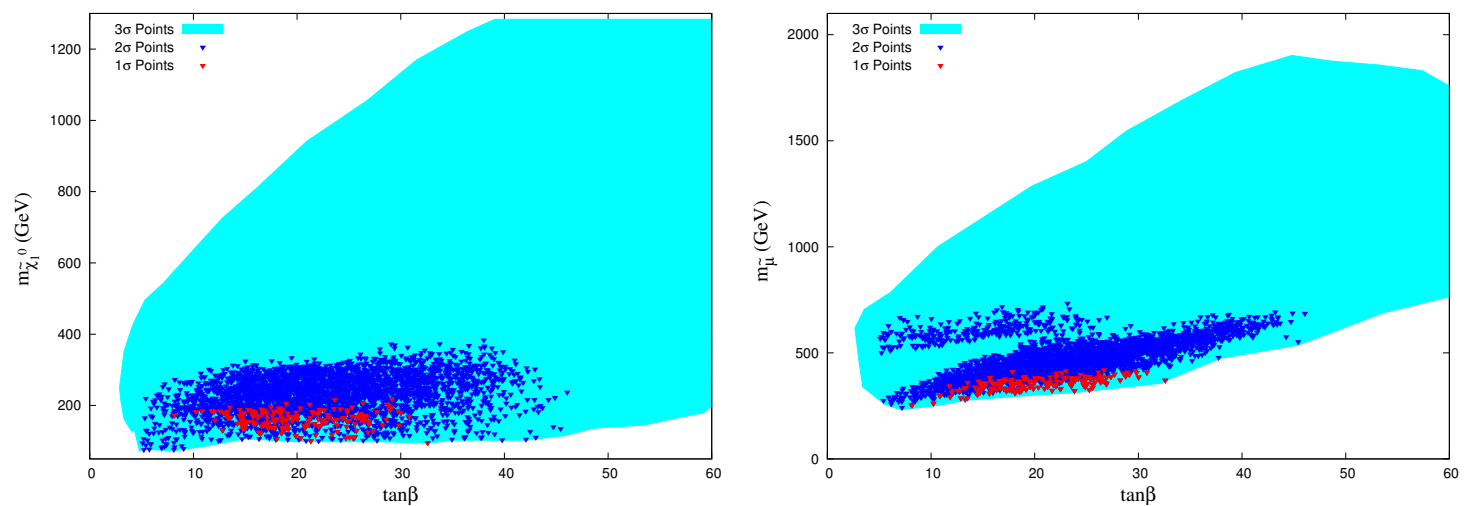

Figure 6. Correlations between low scale parameters: (Left panel) lightest neutralino mass $\left(m_{\tilde{\chi}_{1}^{0}}\right)$ vs $\tan \beta$; (Right panel) smuon mass $\left(m_{\tilde{\mu}}\right)$ vs $\tan \beta$, depending on $[1 \div 3] \sigma$ muon (g-2) excess after satisfying all other constraints discussed in the text. A very similar correlation in $m_{\widetilde{\nu}}-\tan \beta$ plane is found as given in plot on the Right Panel. The slepton and sneutrino mass difference varies from 5-20 GeV, indicating that the sneutrino-wino loop in figure 3 may also contribute significantly.

figure 6 puts an upper limit $(700 \mathrm{GeV})$ on the smuon masses beyond which $2 \sigma$ excess is not found.

Figure 7 shows the correlation between smuon mass and the LSP mass. Since the SUSY contribution to muon (g-2) decreases with the increase of the smuon mass and(or) the LSP masses, we would expect a comet like structure in the smuon-LSP mass plane converging on the lighter side of both the masses, which is reflected in figure 7 (Left). The plot shows only those points for which $\widetilde{\chi}_{1}^{0}$ is the LSP, which has been used as a constraint. Note that, a light $\operatorname{stau}\left(\widetilde{\tau}_{1}\right)$ can also have an impact on the muon (g-2) calculation only if there is a sizeable mixing between smuon and stau states. For our choice of $A_{0}\left(=-2 m_{0}\right)$, we observe that this mixing may vary from negligible amount to as large as $10 \%$ depending on the values of $m_{0}, M_{3}$ and $\tan \beta$. Hence we would expect a sizeable impact of the $\widetilde{\tau}_{1}$ mass in some part of the parameter space, specially, in the low slepton mass regime. Figure 7 (Right) shows the distribution of $m_{\tilde{\tau}_{1}}$ as a function of $m_{\tilde{\chi}_{1}^{0}}$.

\section{Dark matter searches}

\subsubsection{Relic density}

In our analysis, the lightest neutralino $\left(\widetilde{\chi}_{1}^{0}\right)$ is the LSP and DM candidate since we have chosen to work in R-parity conserving scenario. We have checked the compatibility of muon (g-2) allowed parameter space with relic density constraint in figure 8. We consider only those points for which the DM annihilation and(or) co-annihilation are sufficient such that there is no over abundance of the lightest neutralino. As evident from figure 8 , most of the favoured points produce a relic density that is much lower than the present upper limit. In our case, the LSP is composed of either bino or wino or an admixture of both.

Dominant contribution to the DM relic density may come from bulk annihilation of $\widetilde{\chi}_{1}^{0}$ with sleptons (mostly $\widetilde{\tau}_{1}$ ), co-annihilation of $\widetilde{\chi}_{1}^{0}$ with the next to lightest supersymmetric 

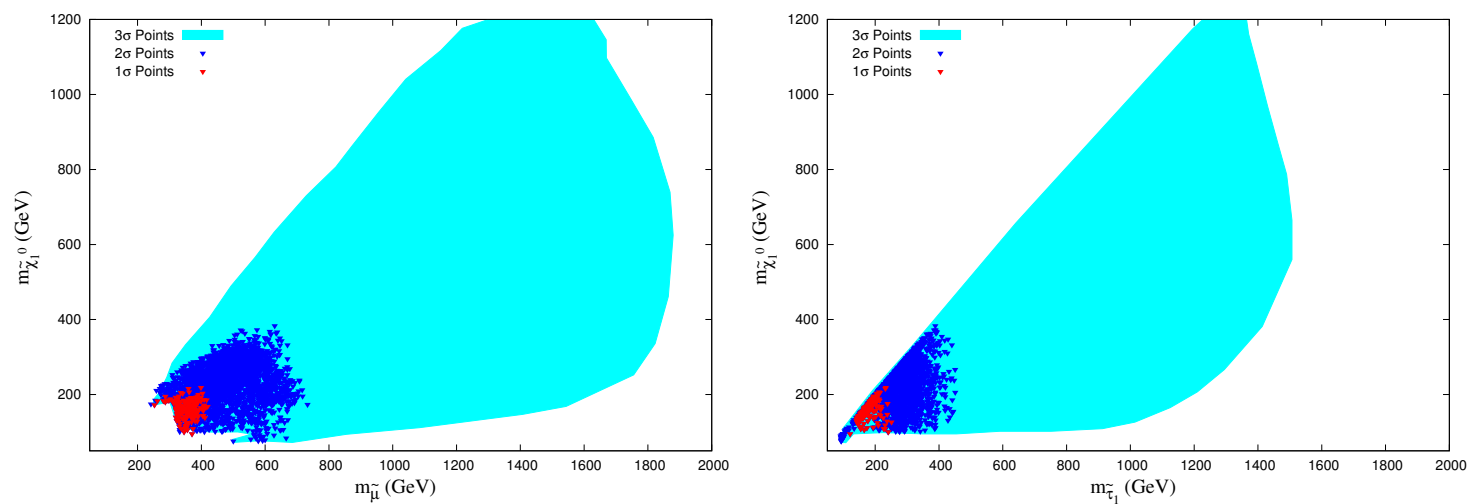

Figure 7. Correlations between low scale parameters: (Left panel) lightest neutralino mass $\left(m_{\widetilde{\chi}_{1}^{0}}\right)$ vs smuon mass $\left(m_{\widetilde{\mu}}\right)$; (Right panel) lightest neutralino mass vs stau mass $\left(m_{\widetilde{\tau}_{1}}\right)$, depending on $[1 \div 3] \sigma$ muon (g-2) excess after satisfying all other constraints discussed in the text.

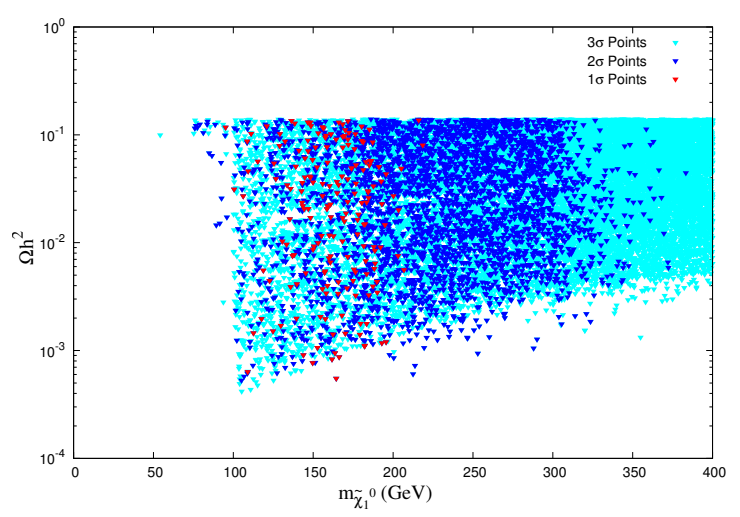

Figure 8. Relic density as a function of the mass of the dark matter, i.e., LSP $\left(\tilde{\chi}_{1}^{0}\right)$ depending on $[1 \div 3] \sigma$ muon (g-2) excess after satisfying all other constraints discussed in the text.

particles (NLSPs) $\widetilde{\tau}_{1}, \widetilde{\chi}_{1}^{ \pm}, \widetilde{\chi}_{2}^{0}$. Thus the muon (g-2) and DM allowed parameter space can be classified into the following categories:

- Bulk annihilation. The pure bulk annihilation region with light sleptons has been ruled out in usual mSUGRA scenario by the LHC constraints. The present exclusion in $m_{0}-m_{1 / 2}$ plane $[95,96]$ is such that the sfermion is always much heavier than the LSP resulting in suppression of slepton mediated DM pair annihilation crosssections. However, with non-universal gaugino and universal scalar masses, we have noted that this can be a possibility and specially when $m_{\widetilde{\tau}_{1}} \leq 130 \mathrm{GeV}$, it plays an important role in keeping $\widetilde{\chi}_{1}^{0}$ abundance under the specified limit. If other charged slepton masses are also close to $m_{\widetilde{\tau}_{1}}$, they can participate in bulk annihilation as well with the final state consisting of two charged leptons. Benchmark points (BP) representing such scenario are illustrated in table 1 (see BP1, BP2).

- Stau co-annihilation. In such scenario, $\widetilde{\tau}_{1}$ is the NLSP and the co-annihilation between $\widetilde{\tau}_{1}$ and LSP is significant to maintain the right relic abundance when $\widetilde{\chi}_{1}^{0}$ is mostly bino-like. Here the $\widetilde{\chi}_{1}^{ \pm}$and(or) $\widetilde{\chi}_{2}^{0}$ states are much heavier. For light $\widetilde{\tau}_{1}$ mass, 


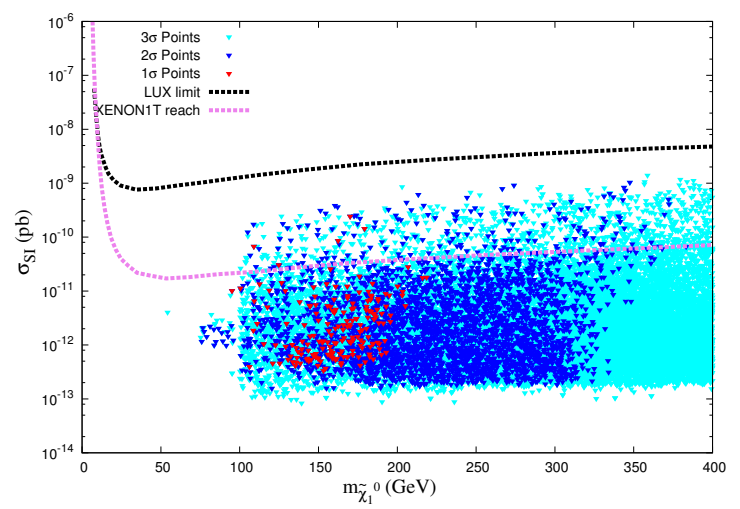

Figure 9. Direct detection cross-section as a function of the mass of the dark matter, i.e., LSP $\left(\tilde{\chi}_{1}^{0}\right)$ depending on $[1 \div 3] \sigma$ muon (g-2) excess after satisfying all other constraints discussed in the text.

dominant contribution comes from bulk annihilation process, whereas $\widetilde{\tau}_{1}-\widetilde{\chi}_{1}^{0}$ coannihilation contribution is roughly $\sim 15-20 \%$ (see BP3 in table 1 ). With relatively larger $m_{\widetilde{\chi}_{1}^{0}}($ see BP4), the co-annihilation processes dominate.

- Chargino co-annihilation. Here, $\widetilde{\chi}_{1}^{ \pm}$and(or) $\widetilde{\chi}_{2}^{0}$ appears as NLSP and consequently $\widetilde{\chi}_{1}^{0}$ annihilation with these sparticles are responsible to determine the relic density assuming the sleptons are much heavier to take part in co-annihilation. When the mass difference between LSP and NLSP is typically $15-25 \mathrm{GeV}$, one obtains PLANCK allowed DM relic abundance (see BP5 and BP6). The final states are usually dominated by quarks and gauge bosons. On the other hand, if $M_{1}$ is much larger than $M_{2}$, the $\widetilde{\chi}_{1}^{ \pm}$and $\widetilde{\chi}_{2}^{0}$ become nearly degenerate with the LSP and they co-annihilate profusely resulting in relic under-abundance.

- Chargino and stau co-annihilation. In such scenarios, $\widetilde{\tau}_{1}, \widetilde{\chi}_{1}^{ \pm}$and(or) $\widetilde{\chi}_{2}^{0}$ masses are close to $m_{\widetilde{\chi}_{1}^{0}}$. Along with bulk annihilation, stau co-annihilation and $\widetilde{\chi}_{1}^{ \pm}$and(or) $\widetilde{\chi}_{2}^{0}$ co-annihilation give rise to correct relic density (see BP7 and BP8 in table 1).

\subsubsection{Direct and indirect detections}

In figure 9 we have shown the distribution of spin independent cross-section $\left(\sigma_{S I}\right)$ as a function of the LSP mass. Note that, all the points shown in these plots obey the relic density upper limit. As evident from the plot, apart from a small part of the $3 \sigma$ allowed region, all the other points appear under the most recent exclusion line provided by LUX on $\sigma_{S I}[130]$ and thus allowed.

The possible contribution to this cross-section comes from $t$-channel $Z$-boson, Higgs, squark mediated diagrams or $s$-channel squark mediated ones. The squarks being heavy do not contribute much. On the other hand, Higgs boson couplings to the first two generation quarks are suppressed. It is well known that within the MSSM framework, the DMnucleon scattering cross-section may increase alarmingly if the LSP consists of a sizeable higgsino component which enhances its coupling with the $Z$-boson. Now in our scenario $\mu \gg M_{1}, M_{2}$ at low scale for most of the parameter space. Hence the LSP state always 
has a negligible higgsino component. In large LSP mass region, the $\mu$ parameter can be comparable with the other gaugino mass parameters and may enhance the scattering cross-section. However, this region of parameter space is not interesting from the $\Delta a_{\mu}$ enhancement viewpoint as discussed earlier. We have also shown in figure 9, the future projected limit of XENON1T [138] experiment. In case of a null result, it will reduce a significant portion of the available parameter space. We have also taken into account the indirect detection cross-section constraints coming from different final states. All our points lie well within the exclusion limits and hence we do not present them in separate plots.

\subsubsection{Some benchmark points}

In this section, we have provided some benchmark points from different regime of the parameter space where $\Delta a_{\mu}, \Omega h^{2}$ and flavour constraints all lie within the $2 \sigma$ ranges of their experimentally measured values. This parameter space is certainly compatible with other collider and low energy constraints mentioned in earlier sections.

In pure bulk (BP1 \& BP2) or stau co-annihilation region (BP3 \& BP4), $m_{\widetilde{\tau}_{1}}$ is relatively light. Since we are working with universal scalar masses at the GUT scale, this implies either the slepton soft masses are light where the smuons are also expected to be light (see e.g. BP2) or $\tan \beta$ is effectively large. Both these scenarios may enhance $\Delta a_{\mu}$ which is reflected in BP1 - BP4. Light chargino may also produce the required enhancement provided the sneutrino masses are not too large. BP5 - BP6 represent chargino ao-annihilation region, where $\widetilde{\chi}_{1}^{ \pm}$is the NLSP. Hence the required enhancement in $\Delta a_{\mu}$ is obtained even with relatively larger smuon mass. BP7 and BP8 have almost degenerate $\widetilde{\tau}_{1}$ and $\widetilde{\chi}_{1}^{ \pm}$masses with large values of $\tan \beta$. Hence the combined effect of light chargino and large $\tan \beta$ produce the required enhancement in $\Delta a_{\mu}$.

A large $\tan \beta$ results in light stau's in the spectrum. For such low scale spectra, from the production and subsequent decays of the gauginos, one would then expect $\tau$-enriched final states associated with missing energy. Since the $\tau$ 's mostly decay hadronically, tagged $\tau$ - jets $+\mathbb{F}_{T}$ signature will be the most suitable one to look for such scenarios. However, the LSP-NLSP mass gap being very small, the $\tau$-jet tagging efficiency will be very small.

The squarks and gluinos are in general heavy in these spectra. Since the gluino mass is heavier than all the squark masses, it can decay into all the squark flavours abundantly giving rise to jets $+\mathbb{F}_{T}$ final state. In the usual mSUGRA scenario, on the other hand, usually the gluino decay via stop into multiple top and neutralinos resulting in the multi $b$-jet final state associated with large missing energy (see, for example, ref. [96]). Moreover, given the present collider exclusion limits, in mSUGRA, with such heavy color sector as in our benchmark scenarios, it is not possible to achieve a slepton sector light enough to enhance the muon (g-2) value to the desired range.

\subsection{Universal gauginos and non-universal scalars — model independent analysis}

In this section, we have discussed the other possible high scale scenario in the present context, namely, universal gauginos with non-universal scalars. 


\begin{tabular}{|c|c|c|c|c|c|c|c|c|}
\hline Parameters & BP1 & BP2 & BP3 & BP4 & BP5 & BP6 & BP7 & BP8 \\
\hline$M_{1}$ & -241.8 & -248.5 & -330.1 & -647.7 & -274.4 & 516.2 & 373.5 & 581.4 \\
$M_{2}$ & 299.9 & -951.1 & -390.0 & 453.1 & 187.2 & -299.3 & 233.5 & 364.3 \\
$M_{3}$ & 1004.1 & 4573.5 & 3075.0 & 1257.9 & 2275.3 & 857.1 & 1396.8 & 2636.8 \\
$m_{0}$ & 325.3 & 168.7 & 346.6 & 386.9 & 428.9 & 483.8 & 301.1 & 465.3 \\
$\tan \beta$ & 29.2 & 5.2 & 16.5 & 30.0 & 22.1 & 29.5 & 21.7 & 26.7 \\
\hline$m_{\widetilde{g}}$ & 2205.5 & 9034.1 & 6269.1 & 2715.8 & 4751.0 & 1911.5 & 3003.5 & 5431.5 \\
$m_{\widetilde{q}_{L}}$ & 1937.9 & 7606.3 & 5327.8 & 2380.4 & 4074.1 & 1724.6 & 2600.7 & 4642.6 \\
$m_{\widetilde{q}_{R}}$ & 1938.6 & 7617.8 & 5345.4 & 2379.3 & 4089.8 & 1726.6 & 2609.4 & 4658.6 \\
$m_{\widetilde{t}_{1}}$ & 1582.8 & 6579.8 & 4594.1 & 1952.8 & 3479.0 & 1339.7 & 2189.8 & 3970.2 \\
$m_{\widetilde{\ell}_{L}}$ & 370.6 & 500.3 & 354.7 & 484.1 & 409.7 & 522.9 & 323.0 & 485.6 \\
$m_{\widetilde{\ell}_{R}}$ & 337.8 & 132.4 & 354.0 & 453.5 & 434.8 & 520.0 & 329.4 & 504.3 \\
$m_{\widetilde{\tau}_{1}}$ & 121.4 & 93.3 & 146.2 & 288.4 & 240.0 & 374.0 & 170.9 & 262.7 \\
$m_{\widetilde{\nu}_{1,2}}$ & 362.3 & 494.7 & 346.2 & 477.8 & 402.2 & 517.0 & 313.4 & 479.4 \\
$m_{\widetilde{\nu}_{3}}$ & 331.8 & 494.2 & 337.8 & 444.4 & 384.5 & 474.0 & 299.8 & 453.4 \\
$\widetilde{\chi}_{1}^{0}$ & 95.2 & 75.9 & 120.9 & 270.6 & 100.6 & 214.1 & 148.6 & 235.0 \\
$\widetilde{\chi}_{2}^{0}$ & 232.1 & 734.1 & 276.2 & 358.1 & 114.0 & 234.7 & 169.0 & 263.4 \\
$\widetilde{\chi}_{1}^{ \pm}$ & 232.1 & 734.1 & 276.2 & 358.1 & 114.0 & 234.7 & 169.0 & 263.4 \\
$\mu$ & 1294.8 & 4863.9 & 3390.9 & 1582.2 & 2637.4 & 1198.1 & 1708.4 & 2990.0 \\
\hline$(g-2)_{\mu} \times 10^{9}$ & 2.64 & 1.82 & 2.28 & 1.42 & 1.54 & 1.34 & 2.64 & 1.51 \\
$\mathrm{BR}(\mathrm{b} \rightarrow \mathrm{s} \gamma) \times 10^{4}$ & 3.18 & 3.31 & 3.31 & 3.24 & 3.31 & 3.11 & 3.30 & 3.30 \\
$\mathrm{BR}\left(\mathrm{B}_{\mathrm{s}} \rightarrow \mu \mu\right) \times 10^{9}$ & 3.81 & 3.07 & 3.07 & 3.38 & 3.08 & 3.74 & 3.12 & 3.09 \\
\hline$\Omega_{S h^{2}} \times 10^{13}(\mathrm{pb})$ & 0.117 & 0.115 & 0.121 & 0.124 & 0.116 & 0.122 & 0.121 & 0.120 \\
$\sigma_{S I}$ & 101.0 & 11.6 & 4.6 & 87.1 & 6.3 & 281.9 & 38.6 & 6.73 \\
\hline
\end{tabular}

Table 1. High scale input parameters and the relevant sparticle masses along with the muon (g-2) value for some of the chosen benchmark points satisfying the collider, DM and low energy constraints discussed in earlier sections. All the mass parameters are written in $\mathrm{GeV}$ unit.

If we assume to have a universal mass parameter $\left(m_{1 / 2}\right)$ for all the gauginos at the high scale, we expect the LSP to be mostly pure bino like at low scale. ${ }^{12}$ This is due to the fact that the renormalisation group evolutions determine the gaugino mass ratios to be $M_{1}: M_{2}: M_{3} \simeq 1: 3: 6$ at low scale. Hence in such scenario, the $\widetilde{\chi}_{1}^{ \pm}$contribution to the loop diagrams will be smaller. However, the bino component can be sufficiently light and can contribute to the enhancement, but then the relic density constraint can be a serious problem as the DM pair annihilation cross-section may not be suitable to satisfy proper relic abundance. The non-universal scalar scenario can be useful as it may

\footnotetext{
${ }^{12}$ We are ignoring the higgsino possibility from the point of our analysis.
} 

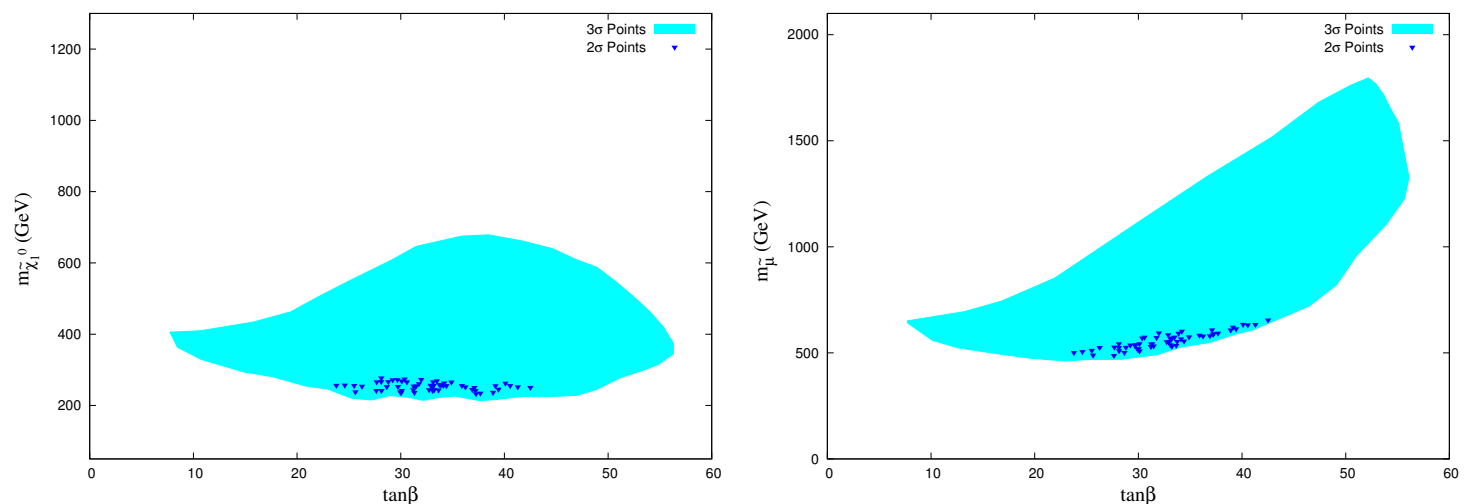

Figure 10. Correlations between low scale parameters: (Left panel) lightest neutralino mass $\left(m_{\tilde{\chi}_{1}^{0}}\right)$ vs $\tan \beta$; (Right panel) smuon mass $\left(m_{\tilde{\mu}}\right)$ vs $\tan \beta$, depending on $[2 \div 3] \sigma$ muon (g-2) excess after satisfying all other constraints discussed in the text. A very similar correlation in $m_{\widetilde{\nu}}$-tan $\beta$ plane is found as given in plot on the Right Panel. We have not found any parameter space in this scenario that satisfy $1 \sigma$ excess.

allow a stau $\left(\widetilde{\tau}_{1}\right)$ to be light enough which can co-annihilate with $\widetilde{\chi}_{1}^{0}$ to make up for the annihilation cross-section. An added advantage of having a bino-LSP is that long-lived or stable chargino constraint which proved to be the most severe in the previous case, will not be applicable here.

In this scenario, the high scale input parameters are following: the universal gaugino mass $\left(m_{1 / 2}\right)$, slepton mass $\left(m_{0}\right)$ and squark mass $\left(m_{0}^{\prime}\right)$. The soft Higgs mass parameters, $m_{H_{d}}$ and $m_{H_{u}}$ are assumed to be equal to $m_{0}$ at the GUT scale. The trilinear coupling, $A_{0}\left(=-2 m_{0}\right)$ is same as before. We note that this scenario can provide $[2 \div 3] \sigma$ excess in $\Delta a_{\mu}$ at its best.

It is evident from figure 10 that both the slepton and LSP masses are heavier in this scenario compared to the earlier case at the low scale. The collider constraints for universal gaugino masses combine to produce these mass limits which prevent from achieving $1 \sigma$ excess of $\Delta a_{\mu}$. Assuming a universal gaugino mass at high scale the gluino appears to be roughly six times heavier than the LSP at low scale. Hence imposing a mass limit of $\sim 1.2 \mathrm{TeV}$ on the gluino mass for heavy squark scenarios automatically implies that the LSP cannot be lighter than $\sim 200 \mathrm{GeV}$. This scenario is being reflected in figure 10 .

There can be another high scale scenario where both the gauginos and scalars are non-universal. For this case, the collider and DM constraints put very similar lower limits on the LSP and slepton masses to that we obtained for the non-universal gaugino and universal scalar scenario. The LSP composition is also very similar. Hence this scenario does not provide any new features to highlight.

\subsection{Non-universal gauginos and universal scalars - model based analysis}

We have discussed the generation of gaugino masses in phenomenological $\mathcal{N}=1$ supergravity scenario in section 2 . We have also noted that if the visible sector possesses unified symmetry, then we need a non-singlet scalar to break that symmetry to achieve the SM gauge group. We have further discussed that in presence of a singlet and a non-singlet 


\begin{tabular}{|c|c|c|}
\hline Model Number & $\begin{array}{c}M_{1}: M_{2}: M_{3} \\
\left(\text { at } \mathrm{M}_{X}\right)\end{array}$ & $\begin{array}{c}\text { Symmetry Breaking } \\
\text { Patterns }\end{array}$ \\
\hline 1 & $-1 / 5:-1: 1$ & $E(6) \stackrel{(35,1) \subset 650}{\longrightarrow}\left(\mathrm{SU}(6) \otimes \mathrm{SU}(2)_{R}\right)$ \\
\hline 2 & $19 / 10: 5 / 2: 1$ & $\mathrm{SO}(10) \stackrel{(1,1) \subset 770}{\longrightarrow}\left(\mathrm{SU}(4) \otimes \mathrm{SU}(2)_{R}\right.$ \\
\hline 3 & $1 / 10:-3 / 2: 1$ & $E(6) \stackrel{(54,0) \subset 650}{\longrightarrow}(\mathrm{SO}(10) \otimes \mathrm{U}(1))_{\text {flipped }}$ \\
\hline 4 & $-1 / 5:-3 / 2: 1$ & $E(6) \stackrel{(210,0) \subset 650,2430}{\longrightarrow}(\mathrm{SO}(10) \otimes \mathrm{U}(1))_{\text {flipped }}$ \\
& $-1 / 2:-3 / 2: 1$ & $\mathrm{SO}(10) \otimes \mathrm{U}(1))_{\text {flipped }} \stackrel{(24) \subset 210}{\longrightarrow} \mathrm{SU}(5)$ \\
\hline 5 & & $\mathrm{SO}(10) \stackrel{(24) \subset 54,210,770}{\longrightarrow} \mathrm{SU}(5)$ \\
& & $\mathrm{SO}(10) \stackrel{(24,0) \subset 54}{\longrightarrow}(\mathrm{SU}(5) \otimes \mathrm{U}(1))_{\text {flipped }}$ \\
& $7 / 10:-3 / 2: 1$ & $\mathrm{SO}(10) \stackrel{(1,1) \subset 54}{\longrightarrow}\left(\mathrm{SU}(4) \otimes \mathrm{SU}(2)_{R}\right)$ \\
\hline 6 & & $\mathrm{SU}(5) \otimes \mathrm{U}(1))_{\text {flipped }}$ \\
\hline
\end{tabular}

Table 2. Gaugino mass models that can explain $[1 \div 3] \sigma$ excess of muon $(\mathrm{g}-2)$. Other models are not compatible with $[1 \div 2] \sigma$ excess within this scheme.

field in the hidden and visible sectors respectively, the generic gaugino mass terms can be written as $M_{i}=M^{\prime}\left[1+\wp \delta_{i}\right]$ (see eq. (2.4)). This function $\wp$ is the ratio of $P, Q$ (see eq. (2.3)). The detail structure of $\wp$ is not easy to reveal. Thus we have encompassed the numerical ranges of $\wp$ which can explain the muon (g-2) excess successfully at $[1 \div 2] \sigma$ level. The MSSM gaugino mass ratios at the high scale is depicted as:

$$
M_{1}: M_{2}: M_{3}=\left(1+\wp \delta_{1}\right):\left(1+\wp \delta_{2}\right):\left(1+\wp \delta_{3}\right)
$$

Earlier in ref. [42], 25 different phenomenological models were analysed under the impression of dark matter search results. There contributions from the singlet scalar was neglected and prime focus was on the non-universal part only. We have revisited those models with their generic structures and from muon (g-2) point of view. Here, we have adjudged those non-universal models and checked which among them can successfully explain $[1 \div 2] \sigma$ excess of $\Delta a_{\mu}$ (see table 2 ). We have analysed these models to find out the ranges of $\wp$ which is a measure of weighted mixing between singlet and non-singlet contributions. Note that among those 25 models (see ref. [42]), only 6 can successfully explain observed muon $(\mathrm{g}-2)$ excess at $[1 \div 2] \sigma$ level after including the singlet contribution. Few models can provide $3 \sigma$ excess but we are not quoting those here. Note that, if this singlet contribution is neglected, all those 25 models fail to explain $\Delta a_{\mu}$ excess at $[1 \div 2] \sigma$ level.

Figure 11 describes the correlation between $M_{1} / M_{3}$ and $\wp$ for different models. There are certain ranges of $\wp$ for which there exist discontinuities in $M_{13}$ which are outcome of the fact that within that range $M_{23}$ or $M_{13}$ vanishes. This happens because both are related for specific models. Around that solution, where $M_{23}$ or $M_{13}$ is very small, no parameter 


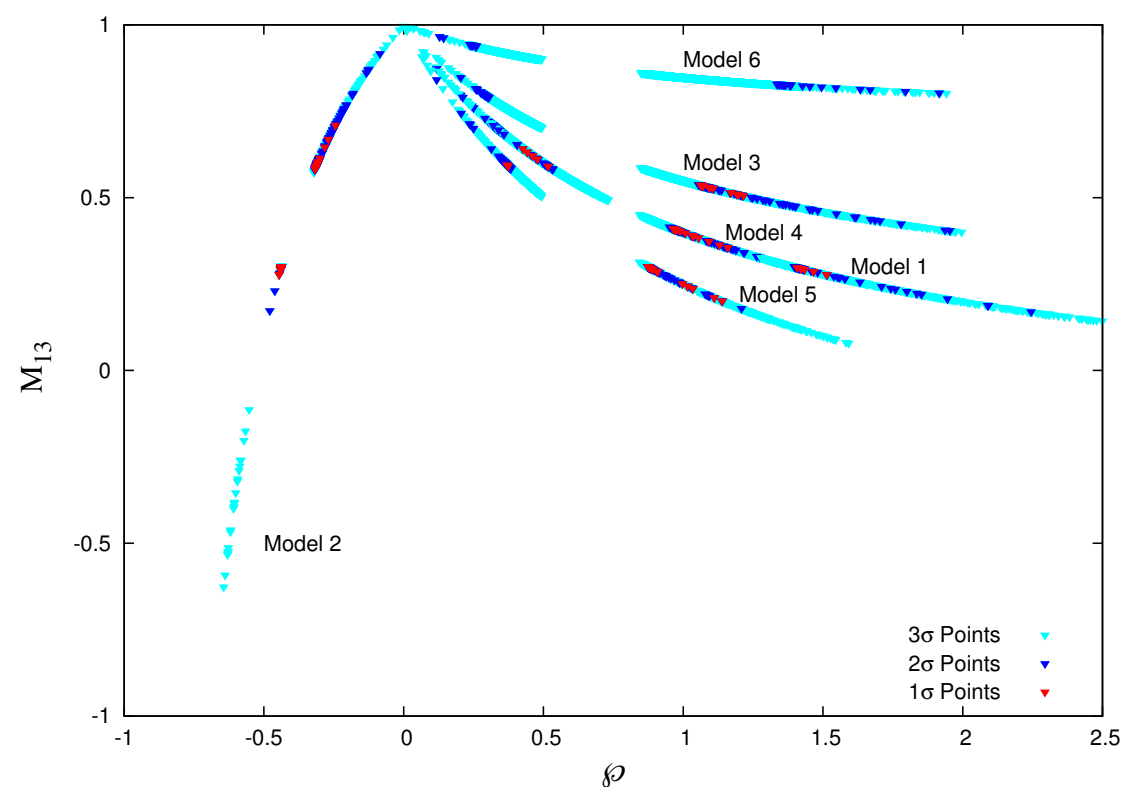

Figure 11. The variations of mixing parameter $\wp$ with $M_{13}$ for different set of models leading to $[1 \div 3] \sigma$ excess in muon (g-2). The other models cannot provide $\Delta a_{\mu}$ excess at level of $[1 \div 2] \sigma$.

space is compatible with other imposed constraints. We have also explicitly shown the dependence of excess of muon (g-2) on the weighted mixing $\wp$.

\section{Conclusion}

High scale supersymmetry breaking is at the center of study since long time due to its minimalistic view. In this paper we have adopted a methodology to understand the correlations among high scale parameters in these type of scenarios, with a goal to explain the $[1 \div 3] \sigma$ excess of muon (g-2) over Standard Model predictions. To enrich the viability of our analysis, we have carefully incorporated the collider constraints without being biased to any particular scenario and also avoided the implementation of just mSUGRA bounds. The experimental constraints coming from simplified model assumptions have been tuned suitably. Hence the constraints imposed in our analysis can be taken over mutatis mutandis.

We have categorized our work in two parts: first we have discussed the model independent correlations among non-universal gaugino masses, and found out the moun (g-2) compatible solutions at $[1 \div 3] \sigma$ level. We observe that none of the existing models' predictions fit within this. Thus from muon (g-2) point of view, these models are not capable enough. We have further analysed the high scale parameter space in terms of the low scale masses of sleptons, smuons, lighter chargino and lightest neutralino (LSP), along with $\tan \beta$. Then we have also scrutinized such parameter space in the light of direct and indirect searches for DM. We have encompassed that regime by showing the dependence of relic density and spin dependent cross-section $\left(\sigma_{S I}\right)$ on mass of the DM.

We have briefly discussed the general structure of the gaugino masses that include the contributions from singlet and non-singlet chiral super-fields. Thus in such cases there is an 
extra parameter which is a measure of mixing of contributions from both fields. We have stuck to minimal models, i.e., restricted to one non-singlet field and explored the range of that mixing parameter from muon (g-2) viewpoint along with other constraints. We have found only few of the existing models can explain the excess in $\Delta a_{\mu}$ at $[1 \div 2] \sigma$ level.

In summary, we have captured the model independent features of non-universality considering muon (g-2) excess on a serious note. This broadly classified picture is also grabbed for some particular cases in terms of some specific benchmark points.

\section{Acknowledgments}

Work of JC is supported by Department of Science \& Technology, Government of INDIA under the Grant Agreement number IFA12-PH-34 (INSPIRE Faculty Award). The work of AC, SM was partially supported by funding available from the Department of Atomic Energy, Government of India, for the Regional Centre for Accelerator-based Particle Physics (RECAPP), Harish-Chandra Research Institute.

Open Access. This article is distributed under the terms of the Creative Commons Attribution License (CC-BY 4.0), which permits any use, distribution and reproduction in any medium, provided the original author(s) and source are credited.

\section{References}

[1] E. Witten, Is supersymmetry really broken?, Int. J. Mod. Phys. A 10 (1995) 1247 [hep-th/9409111] [INSPIRE].

[2] E. Witten, Dynamical Breaking of Supersymmetry, Nucl. Phys. B 188 (1981) 513 [InSPIRE].

[3] E. Witten, Lecture Notes On Supersymmetry, (1981) [INSPIRE].

[4] E. Witten, Constraints on Supersymmetry Breaking, Nucl. Phys. B 202 (1982) 253 [INSPIRE].

[5] S. Dimopoulos and H. Georgi, Softly Broken Supersymmetry and SU(5), Nucl. Phys. B 193 (1981) 150 [INSPIRE].

[6] A.E. Nelson and N. Seiberg, $R$ symmetry breaking versus supersymmetry breaking, Nucl. Phys. B 416 (1994) 46 [hep-ph/9309299] [INSPIRE].

[7] K.A. Intriligator, N. Seiberg and D. Shih, Dynamical SUSY breaking in meta-stable vacua, JHEP 04 (2006) 021 [hep-th/0602239] [INSPIRE].

[8] K.A. Intriligator, N. Seiberg and D. Shih, Supersymmetry breaking, R-symmetry breaking and metastable vacua, JHEP 07 (2007) 017 [hep-th/0703281] [INSPIRE].

[9] K.A. Intriligator and N. Seiberg, Lectures on Supersymmetry Breaking, Class. Quant. Grav. 24 (2007) S741 [hep-ph/0702069] [INSPIRE].

[10] S. Ferrara, J. Scherk and B. Zumino, Algebraic Properties of Extended Supergravity Theories, Nucl. Phys. B 121 (1977) 393 [INSPIRE].

[11] E. Cremmer and J. Scherk, Algebraic Simplifications in Supergravity Theories, Nucl. Phys. B 127 (1977) 259 [INSPIRE]. 
[12] E. Cremmer, J. Scherk and S. Ferrara, U(n) Invariance in Extended Supergravity, Phys. Lett. B 68 (1977) 234 [INSPIRE].

[13] E. Cremmer et al., Super-Higgs effect in supergravity with general scalar interactions, Phys. Lett. B 79 (1978) 231 [INSPIRE].

[14] E. Cremmer et al., Spontaneous Symmetry Breaking and Higgs Effect in Supergravity Without Cosmological Constant, Nucl. Phys. B 147 (1979) 105 [inSPIRE].

[15] E. Cremmer, S. Ferrara, L. Girardello and A. Van Proeyen, Coupling Supersymmetric Yang-Mills Theories to Supergravity, Phys. Lett. B 116 (1982) 231 [INSPIRE].

[16] E. Cremmer, S. Ferrara, L. Girardello and A. Van Proeyen, Yang-Mills Theories with Local Supersymmetry: Lagrangian, Transformation Laws and SuperHiggs Effect, Nucl. Phys. B 212 (1983) 413 [INSPIRE].

[17] L. Álvarez-Gaumé, J. Polchinski and M.B. Wise, Minimal Low-Energy Supergravity, Nucl. Phys. B 221 (1983) 495 [INSPIRE].

[18] H.P. Nilles, Supersymmetry, Supergravity and Particle Physics, Phys. Rept. 110 (1984) 1 [INSPIRE].

[19] J.D. Lykken, Introduction to supersymmetry, hep-th/9612114 [INSPIRE].

[20] L.J. Hall, J.D. Lykken and S. Weinberg, Supergravity as the Messenger of Supersymmetry Breaking, Phys. Rev. D 27 (1983) 2359 [INSPIRE].

[21] R. Barbieri, S. Ferrara and C.A. Savoy, Gauge Models with Spontaneously Broken Local Supersymmetry, Phys. Lett. B 119 (1982) 343 [INSPIRE].

[22] A.H. Chamseddine, R.L. Arnowitt and P. Nath, Locally Supersymmetric Grand Unification, Phys. Rev. Lett. 49 (1982) 970 [INSPIRE].

[23] N. Ohta, Grand unified theories based on local supersymmetry, Prog. Theor. Phys. 70 (1983) 542 [INSPIRE].

[24] J.R. Ellis, D.V. Nanopoulos and K. Tamvakis, Grand Unification in Simple Supergravity, Phys. Lett. B 121 (1983) 123 [INSPIRE].

[25] L.E. Ibáñez, Locally Supersymmetric SU(5) Grand Unification, Phys. Lett. B 118 (1982) 73 [INSPIRE].

[26] K. Inoue, A. Kakuto, H. Komatsu and S. Takeshita, Aspects of Grand Unified Models with Softly Broken Supersymmetry, Prog. Theor. Phys. 68 (1982) 927 [InSPIRE].

[27] L.E. Ibáñez and G.G. Ross, SU(2)-L $x$ U(1) Symmetry Breaking as a Radiative Effect of Supersymmetry Breaking in Guts, Phys. Lett. B 110 (1982) 215 [INSPIRE].

[28] J.R. Ellis, K. Enqvist, D.V. Nanopoulos and K. Tamvakis, Gaugino Masses and Grand Unification, Phys. Lett. B 155 (1985) 381 [INSPIRE].

[29] M. Drees, Phenomenological Consequences of $N=1$ Supergravity Theories With Nonminimal Kinetic Energy Terms for Vector Superfields, Phys. Lett. B 158 (1985) 409 [INSPIRE].

[30] J.R. Ellis, C. Kounnas and D.V. Nanopoulos, No Scale Supersymmetric Guts, Nucl. Phys. B 247 (1984) 373 [INSPIRE].

[31] J. Chakrabortty and A. Raychaudhuri, Dimension-5 operators and the unification condition in $\mathrm{SO}(10)$ and $E_{6}$, arXiv: 1006.1252 [InSPIRE]. 
[32] S. Bhattacharya and J. Chakrabortty, Gaugino mass non-universality in an $\mathrm{SO}(10)$ supersymmetric Grand Unified Theory: Low-energy spectra and collider signals, Phys. Rev. D 81 (2010) 015007 [arXiv:0903.4196] [INSPIRE].

[33] S.P. Martin, Non-universal gaugino masses from non-singlet F-terms in non-minimal unified models, Phys. Rev. D 79 (2009) 095019 [arXiv:0903.3568] [InSPIRE].

[34] J. Chakrabortty and A. Raychaudhuri, A note on dimension-5 operators in GUTs and their impact, Phys. Lett. B 673 (2009) 57 [arXiv:0812.2783] [INSPIRE].

[35] T. Li and D.V. Nanopoulos, Generalizing Minimal Supergravity, Phys. Lett. B 692 (2010) 121 [arXiv: 1002 .4183] [INSPIRE].

[36] M. Atkins and X. Calmet, Unitarity bounds on low scale quantum gravity, Eur. Phys. J. C 70 (2010) 381 [arXiv: 1005.1075] [INSPIRE].

[37] C. Balázs, T. Li, D.V. Nanopoulos and F. Wang, Supersymmetry Breaking Scalar Masses and Trilinear Soft Terms in Generalized Minimal Supergravity, JHEP 09 (2010) 003 [arXiv: 1006.5559] [INSPIRE].

[38] F. Wang, W. Wang and J.M. Yang, A split SUSY model from SUSY GUT, JHEP 03 (2015) 050 [arXiv: 1501.02906] [INSPIRE].

[39] M. Chakraborti, U. Chattopadhyay, S. Rao and D.P. Roy, Higgsino Dark Matter in Nonuniversal Gaugino Mass Models, Phys. Rev. D 91 (2015) 035022 [arXiv:1411.4517] [INSPIRE].

[40] D.J. Miller and A.P. Morais, Supersymmetric $\mathrm{SO}(10)$ Grand Unification at the LHC and Beyond, JHEP 12 (2014) 132 [arXiv:1408.3013] [INSPIRE].

[41] S.P. Martin, Nonuniversal gaugino masses and seminatural supersymmetry in view of the Higgs boson discovery, Phys. Rev. D 89 (2014) 035011 [arXiv: 1312.0582] [INSPIRE].

[42] J. Chakrabortty, S. Mohanty and S. Rao, Non-universal gaugino mass GUT models in the light of dark matter and LHC constraints, JHEP 02 (2014) 074 [arXiv:1310.3620] [INSPIRE].

[43] S. Bhattacharya, S. Chakdar, K. Ghosh and S. Nandi, Non-universal SUGRA at LHC: Prospects and Discovery Potential, Phys. Rev. D 89 (2014) 015004 [arXiv:1309.0036] [INSPIRE].

[44] D.J. Miller and A.P. Morais, Supersymmetric SU(5) Grand Unification for a Post Higgs Boson Era, JHEP 10 (2013) 226 [arXiv:1307.1373] [INSPIRE].

[45] J.E. Younkin and S.P. Martin, Non-universal gaugino masses, the supersymmetric little hierarchy problem and dark matter, Phys. Rev. D 85 (2012) 055028 [arXiv:1201.2989] [INSPIRE].

[46] S.F. King, J.P. Roberts and D.P. Roy, Natural dark matter in SUSY GUTs with non-universal gaugino masses, JHEP 10 (2007) 106 [arXiv:0705.4219] [INSPIRE].

[47] A. Corsetti and P. Nath, Gaugino mass nonuniversality and dark matter in SUGRA, strings and D-brane models, Phys. Rev. D 64 (2001) 125010 [hep-ph/0003186] [INSPIRE].

[48] U. Chattopadhyay and P. Nath, $b-\tau$ unification, $g_{\mu}-2$, the $b \rightarrow s+\gamma$ constraint and nonuniversalities, Phys. Rev. D 65 (2002) 075009 [hep-ph/0110341] [INSPIRE].

[49] G. Anderson, H. Baer, C.-h. Chen and X. Tata, The Reach of Fermilab Tevatron upgrades for SU(5) supergravity models with nonuniversal gaugino masses, Phys. Rev. D 61 (2000) 095005 [hep-ph/9903370] [INSPIRE]. 
[50] K. Huitu, Y. Kawamura, T. Kobayashi and K. Puolamaki, Phenomenological constraints on SUSY SU(5) GUTs with nonuniversal gaugino masses, Phys. Rev. D 61 (2000) 035001 [hep-ph/9903528] [INSPIRE].

[51] K. Huitu et al., Search for Higgs Bosons in SUSY Cascades in CMS and Dark Matter with Non-universal Gaugino Masses, Eur. Phys. J. C 58 (2008) 591 [arXiv:0808.3094] [INSPIRE].

[52] U. Chattopadhyay, D. Das and D.P. Roy, Mixed Neutralino Dark Matter in Nonuniversal Gaugino Mass Models, Phys. Rev. D 79 (2009) 095013 [arXiv:0902.4568] [InSPIRE].

[53] S.P. Das, M. Guchait and D.P. Roy, Testing SUSY models for the muon g-2 anomaly via chargino-neutralino pair production at the LHC, Phys. Rev. D 90 (2014) 055011 [arXiv:1406.6925] [INSPIRE].

[54] A. Kaminska, G.G. Ross and K. Schmidt-Hoberg, Non-universal gaugino masses and fine tuning implications for SUSY searches in the MSSM and the GNMSSM, JHEP 11 (2013) 209 [arXiv: 1308.4168] [INSPIRE].

[55] S. Bhattacharya, A. Datta and B. Mukhopadhyaya, Non-universal gaugino masses: A signal-based analysis for the Large Hadron Collider, JHEP 10 (2007) 080 [arXiv: 0708.2427] [INSPIRE].

[56] H. Abe, T. Kobayashi and Y. Omura, Relaxed fine-tuning in models with non-universal gaugino masses, Phys. Rev. D 76 (2007) 015002 [hep-ph/0703044] [INSPIRE].

[57] S. Khalil, Nonuniversal gaugino phases and the LSP relic density, Phys. Lett. B 484 (2000) 98 [hep-ph/9910408] [INSPIRE].

[58] S. Chakrabarti, A. Datta and N. Mondal, Probing non-universal gaugino masses: Prospects at the Tevatron, Pramana 63 (2004) 1355.

[59] A. Birkedal-Hansen and B.D. Nelson, Relic neutralino densities and detection rates with nonuniversal gaugino masses, Phys. Rev. D 67 (2003) 095006 [hep-ph/0211071] [InSPIRE].

[60] D.G. Cerdeno and C. Muñoz, Neutralino dark matter in supergravity theories with non-universal scalar and gaugino masses, JHEP 10 (2004) 015 [hep-ph/0405057] [INSPIRE].

[61] S.I. Bityukov and N.V. Krasnikov, Search for SUSY at LHC in jets $+E_{T}^{\mathrm{miss}}$ final states for the case of nonuniversal gaugino masses, Phys. Lett. B 469 (1999) 149 [hep-ph/9907257] [INSPIRE].

[62] S.I. Bityukov and N.V. Krasnikov, Gaugino pair production at LHC for the case of nonuniversal gaugino masses, Nuovo Cim. A 112 (1999) 913 [hep-ph/9903519] [INSPIRE].

[63] S.I. Bityukov and N.V. Krasnikov, The LHC (CMS) discovery potential for models with effective supersymmetry and nonuniversal gaugino masses, Phys. Atom. Nucl. 65 (2002) 1341 [hep-ph/0102179] [INSPIRE].

[64] S.I. Bityukov and N.V. Krasnikov, LHC (CMS) SUSY discovery potential for nonuniversal gaugino and squark masses and the determination of the effective SUSY scale, hep-ph/0210269 [INSPIRE].

[65] Muon G-2 collaboration, G.W. Bennett et al., Final Report of the Muon E821 Anomalous Magnetic Moment Measurement at BNL, Phys. Rev. D 73 (2006) 072003 [hep-ex/0602035] [INSPIRE]. 
[66] B.L. Roberts, Status of the Fermilab Muon $(g-2)$ Experiment, Chin. Phys. C 34 (2010) 741 [arXiv: 1001.2898 ] [INSPIRE].

[67] A. Nyffeler, Status of hadronic light-by-light scattering in the muon g-2, Nuovo Cim. C 037 (2014) 173 [arXiv:1312.4804] [INSPIRE].

[68] D.A. Kosower, L.M. Krauss and N. Sakai, Low-Energy Supergravity and the Anomalous Magnetic Moment of the Muon, Phys. Lett. B 133 (1983) 305 [inSPIRE].

[69] T.C. Yuan, R.L. Arnowitt, A.H. Chamseddine and P. Nath, Supersymmetric Electroweak Effects on G-2 (mu), Z. Phys. C 26 (1984) 407 [InSPIRE].

[70] H. Fargnoli, C. Gnendiger, S. Paßehr, D. Stöckinger and H. Stöckinger-Kim, Two-loop corrections to the muon magnetic moment from fermion/sfermion loops in the MSSM: detailed results, JHEP 02 (2014) 070 [arXiv:1311.1775] [INSPIRE].

[71] H.G. Fargnoli, C. Gnendiger, S. Paßehr, D. Stöckinger and H. Stöckinger-Kim, Non-decoupling two-loop corrections to $(g-2)_{\mu}$ from fermion/sfermion loops in the MSSM, Phys. Lett. B 726 (2013) 717 [arXiv:1309.0980] [INSPIRE].

[72] T. Moroi, The muon anomalous magnetic dipole moment in the minimal supersymmetric standard model, Phys. Rev. D 53 (1996) 6565 [hep-ph/9512396] [INSPIRE].

[73] S. Heinemeyer, D. Stöckinger and G. Weiglein, Two loop SUSY corrections to the anomalous magnetic moment of the muon, Nucl. Phys. B 690 (2004) 62 [hep-ph/0312264] [InSPIRE].

[74] D. Stöckinger, The Muon Magnetic Moment and Supersymmetry, J. Phys. G 34 (2007) R45 [hep-ph/0609168] [INSPIRE].

[75] G.-C. Cho, K. Hagiwara, Y. Matsumoto and D. Nomura, The MSSM confronts the precision electroweak data and the muon g-2, JHEP 11 (2011) 068 [arXiv:1104.1769] [INSPIRE].

[76] S. Akula and P. Nath, Gluino-driven radiative breaking, Higgs boson mass, muon g-2 and the Higgs diphoton decay in supergravity unification, Phys. Rev. D 87 (2013) 115022 [arXiv: 1304.5526] [INSPIRE].

[77] M. Endo, K. Hamaguchi, T. Kitahara and T. Yoshinaga, Probing Bino contribution to muon g-2, JHEP 11 (2013) 013 [arXiv:1309.3065] [INSPIRE].

[78] M. Endo, K. Hamaguchi, S. Iwamoto and T. Yoshinaga, Muon g-2 vs LHC in Supersymmetric Models, JHEP 01 (2014) 123 [arXiv:1303.4256] [INSPIRE].

[79] M. Chakraborti, U. Chattopadhyay, A. Choudhury, A. Datta and S. Poddar, The Electroweak Sector of the pMSSM in the Light of LHC $-8 \mathrm{TeV}$ and Other Data, JHEP 07 (2014) 019 [arXiv: 1404.4841] [INSPIRE].

[80] J.L. Lopez, D.V. Nanopoulos and X. Wang, Large (g-2)-mu in $\mathrm{SU}(5) \times \mathrm{U}(1)$ supergravity models, Phys. Rev. D 49 (1994) 366 [hep-ph/9308336] [INSPIRE].

[81] U. Chattopadhyay and P. Nath, Probing supergravity grand unification in the Brookhaven g-2 experiment, Phys. Rev. D 53 (1996) 1648 [hep-ph/9507386] [INSPIRE].

[82] U. Chattopadhyay and P. Nath, Upper limits on sparticle masses from g-2 and the possibility for discovery of SUSY at colliders and in dark matter searches, Phys. Rev. Lett. 86 (2001) 5854 [hep-ph/0102157] [INSPIRE].

[83] K.S. Babu, I. Gogoladze, Q. Shafi and C.S. Ün, Muon g-2, 125 GeV Higgs boson and neutralino dark matter in a flavor symmetry-based MSSM, Phys. Rev. D 90 (2014) 116002 [arXiv: 1406.6965] [INSPIRE]. 
[84] S. Mohanty, S. Rao and D.P. Roy, Reconciling the muon $g-2$ and dark matter relic density with the LHC results in nonuniversal gaugino mass models, JHEP 09 (2013) 027 [arXiv:1303.5830] [INSPIRE].

[85] I. Gogoladze, F. Nasir, Q. Shafi and C.S. Un, Nonuniversal Gaugino Masses and Muon g-2, Phys. Rev. D 90 (2014) 035008 [arXiv:1403.2337] [inSPIRE].

[86] M. Adeel Ajaib, I. Gogoladze and Q. Shafi, GUT-inspired supersymmetric model for $h \rightarrow \gamma \gamma$ and the muon g-2, Phys. Rev. D 91 (2015) 095005 [arXiv:1501.04125] [InSPIRE].

[87] LEP2 SUSY working group, http://lepsusy.web.cern.ch/lepsusy/.

[88] ATLAS collaboration, Observation of a new particle in the search for the Standard Model Higgs boson with the ATLAS detector at the LHC, Phys. Lett. B 716 (2012) 1 [arXiv: 1207.7214] [INSPIRE].

[89] CMS collaboration, Observation of a new boson at a mass of $125 \mathrm{GeV}$ with the CMS experiment at the LHC, Phys. Lett. B 716 (2012) 30 [arXiv:1207.7235] [INSPIRE].

[90] ATLAS collaboration, Search for direct production of charginos and neutralinos in events with three leptons and missing transverse momentum in $\sqrt{s}=8 T e V$ pp collisions with the ATLAS detector, JHEP 04 (2014) 169 [arXiv: 1402.7029] [INSPIRE].

[91] CMS collaboration, Searches for electroweak production of charginos, neutralinos and sleptons decaying to leptons and $W, Z$ and Higgs bosons in pp collisions at $8 \mathrm{TeV}$, Eur. Phys. J. C 74 (2014) 3036 [arXiv:1405.7570] [INSPIRE].

[92] M. Chakraborti, U. Chattopadhyay, A. Choudhury, A. Datta and S. Poddar, Reduced LHC constraints for higgsino-like heavier electroweakinos, arXiv:1507.01395 [INSPIRE].

[93] ATLAS collaboration, Search for direct pair production of a chargino and a neutralino decaying to the $125 \mathrm{GeV}$ Higgs boson in $\sqrt{s}=8 \mathrm{TeV}$ pp collisions with the ATLAS detector, Eur. Phys. J. C 75 (2015) 208 [arXiv:1501.07110] [INSPIRE].

[94] ATLAS collaboration, Search for direct production of charginos, neutralinos and sleptons in final states with two leptons and missing transverse momentum in pp collisions at $\sqrt{s}=8 \mathrm{TeV}$ with the ATLAS detector, JHEP 05 (2014) 071 [arXiv:1403.5294] [INSPIRE].

[95] ATLAS collaboration, Search for squarks and gluinos with the ATLAS detector in final states with jets and missing transverse momentum using $\sqrt{s}=8$ TeV proton-proton collision data, JHEP 09 (2014) 176 [arXiv:1405.7875] [INSPIRE].

[96] ATLAS collaboration, Search for strong production of supersymmetric particles in final states with missing transverse momentum and at least three b-jets at $\sqrt{s}=8 \mathrm{TeV}$ proton-proton collisions with the ATLAS detector, JHEP 1410 (2014) 24 [arXiv:1407.0600] [INSPIRE].

[97] T.J. LeCompte and S.P. Martin, Compressed supersymmetry after 1/fb at the Large Hadron Collider, Phys. Rev. D 85 (2012) 035023 [arXiv:1111.6897] [InSPIRE].

[98] B. Bhattacherjee and K. Ghosh, Degenerate SUSY search at the $8 \mathrm{TeV} L H C$, arXiv:1207.6289 [INSPIRE].

[99] H.K. Dreiner, M. Krämer and J. Tattersall, How low can SUSY go? Matching, monojets and compressed spectra, Europhys. Lett. 99 (2012) 61001 [arXiv:1207.1613] [INSPIRE].

[100] B. Bhattacherjee, A. Choudhury, K. Ghosh and S. Poddar, Compressed supersymmetry at $14 \mathrm{TeV}$ LHC, Phys. Rev. D 89 (2014) 037702 [arXiv:1308.1526] [InSPIRE]. 
[101] S. Mukhopadhyay, M.M. Nojiri and T.T. Yanagida, Compressed SUSY search at the $13 \mathrm{TeV}$ LHC using kinematic correlations and structure of ISR jets, JHEP 1410 (2014) 12 [arXiv: 1403.6028] [INSPIRE].

[102] CMS collaboration, Summary of cms susy results in sms framework, https://twiki.cern.ch/twiki/pub/CMSPublic/SUSYSMSSummaryPlots8TeV/barplot ICHEP2014.pdf.

[103] ATLAS, Summary of atlas susy searches, https://atlas.web.cern.ch/Atlas/GROUPS/PHYSICS/CombinedSummaryPlots/SUSY/ ATLAS_SUSY_Summary/ATLAS_SUSY_Summary.png.

[104] ATLAS collaboration, Search for top squark pair production in final states with one isolated lepton, jets and missing transverse momentum in $\sqrt{s}=8 \mathrm{TeV} p p$ collisions with the ATLAS detector, JHEP 11 (2014) 118 [arXiv: 1407.0583] [INSPIRE].

[105] ATLAS collaboration, Search for direct pair production of the top squark in all-hadronic final states in proton-proton collisions at $\sqrt{s}=8 \mathrm{TeV}$ with the ATLAS detector, JHEP 09 (2014) 015 [arXiv: 1406.1122] [inSPIRE].

[106] ATLAS collaboration, Search for direct top-squark pair production in final states with two leptons in pp collisions at $\sqrt{s}=8 \mathrm{TeV}$ with the ATLAS detector, JHEP 06 (2014) 124 [arXiv: 1403.4853] [INSPIRE].

[107] ATLAS collaboration, Search for pair-produced third-generation squarks decaying via charm quarks or in compressed supersymmetric scenarios in pp collisions at $\sqrt{s}=8 \mathrm{TeV}$ with the ATLAS detector, Phys. Rev. D 90 (2014) 052008 [arXiv:1407.0608] [INSPIRE].

[108] ATLAS collaboration, Search for direct third-generation squark pair production in final states with missing transverse momentum and two b-jets in $\sqrt{s}=8$ TeV pp collisions with the ATLAS detector, JHEP 10 (2013) 189 [arXiv:1308.2631] [INSPIRE].

[109] C.H. Chen, M. Drees and J.F. Gunion, A nonstandard string/SUSY scenario and its phenomenological implications, Phys. Rev. D 55 (1997) 330 [hep-ph/9607421] [INSPIRE].

[110] CMS collaboration, Search for disappearing tracks in proton-proton collisions at $\sqrt{s}=8 \mathrm{TeV}, \mathrm{JHEP} 01$ (2015) 096 [arXiv:1411.6006] [INSPIRE].

[111] ATLAS collaboration, Search for charginos nearly mass degenerate with the lightest neutralino based on a disappearing-track signature in pp collisions at $\sqrt{s}=8 \mathrm{TeV}$ with the ATLAS detector, Phys. Rev. D 88 (2013) 112006 [arXiv:1310.3675] [INSPIRE].

[112] ATLAS collaboration, Searches for heavy long-lived charged particles with the ATLAS detector in proton-proton collisions at $\sqrt{s}=8 \mathrm{TeV}$, JHEP 01 (2015) 068 [arXiv: 1411.6795] [INSPIRE].

[113] CMS collaboration, Search for decays of stopped long-lived particles produced in proton-proton collisions at $\sqrt{s}=8 \mathrm{TeV}$, Eur. Phys. J. C 75 (2015) 151 [arXiv: 1501.05603] [INSPIRE].

[114] CMS collaboration, Reinterpreting the results of the search for long-lived charged particles in the pMSSM and other BSM scenarios, Cms-pas-exo-13-006 (Reinterpreting the results of the search for long-lived charged particles in the pMSSM and other BSM scenarios).

[115] BaBar collaboration, J.P. Lees et al., Precision Measurement of the $B \rightarrow X_{s} \gamma$ Photon Energy Spectrum, Branching Fraction and Direct CP Asymmetry $A_{C P}\left(B \rightarrow X_{s+d} \gamma\right)$, Phys. Rev. Lett. 109 (2012) 191801 [arXiv:1207.2690] [INSPIRE]. 
[116] LHCb collaboration, Measurement of the $B_{s}^{0} \rightarrow \mu^{+} \mu^{-}$branching fraction and search for $B^{0} \rightarrow \mu^{+} \mu^{-}$decays at the LHCb experiment, Phys. Rev. Lett. 111 (2013) 101805 [arXiv:1307.5024] [INSPIRE].

[117] CMS collaboration, Measurement of the $B_{s}^{0} \rightarrow \mu^{+} \mu^{-}$branching fraction and search for $B^{0} \rightarrow \mu^{+} \mu^{-}$with the CMS Experiment, Phys. Rev. Lett. 111 (2013) 101804 [arXiv:1307.5025] [INSPIRE].

[118] WMAP collaboration, G. Hinshaw et al., Nine-Year Wilkinson Microwave Anisotropy Probe (WMAP) Observations: Cosmological Parameter Results, Astrophys. J. Suppl. 208 (2013) 19 [arXiv:1212.5226] [INSPIRE].

[119] Planck collaboration, P.A.R. Ade et al., Planck 2013 results. XVI. Cosmological parameters, Astron. Astrophys. 571 (2014) A16 [arXiv:1303.5076] [INSPIRE].

[120] Z.G. Berezhiani and M.Y. Khlopov, Cosmology of Spontaneously Broken Gauge Family Symmetry, Z. Phys. C 49 (1991) 73 [inSPIRE].

[121] C. Boehm, P. Fayet and J. Silk, Light and heavy dark matter particles, Phys. Rev. D 69 (2004) 101302 [hep-ph/0311143] [INSPIRE].

[122] E. Ma, Supersymmetric Model of Radiative Seesaw Majorana Neutrino Masses, Annales Fond. Broglie 31 (2006) 285 [hep-ph/0607142] [INSPIRE].

[123] P.-H. Gu, Unified mass origin at TeV for dark matter and Dirac neutrinos, Phys. Lett. B 661 (2008) 290 [arXiv:0710.1044] [INSPIRE].

[124] T. Hur, H.-S. Lee and S. Nasri, A supersymmetric U(1)-prime model with multiple dark matters, Phys. Rev. D 77 (2008) 015008 [arXiv:0710.2653] [INSPIRE].

[125] Q.-H. Cao, E. Ma, J. Wudka and C.-P. Yuan, Multipartite dark matter, arXiv:0711.3881 [INSPIRE].

[126] T. Hambye, Hidden vector dark matter, JHEP 01 (2009) 028 [arXiv:0811.0172] [INSPIRE].

[127] M. Aoki, M. Duerr, J. Kubo and H. Takano, Multi-Component Dark Matter Systems and Their Observation Prospects, Phys. Rev. D 86 (2012) 076015 [arXiv:1207.3318] [INSPIRE].

[128] J. Heeck and H. Zhang, Exotic Charges, Multicomponent Dark Matter and Light Sterile Neutrinos, JHEP 05 (2013) 164 [arXiv:1211.0538] [INSPIRE].

[129] XENON100 collaboration, E. Aprile et al., Dark Matter Results from 225 Live Days of XENON100 Data, Phys. Rev. Lett. 109 (2012) 181301 [arXiv:1207.5988] [INSPIRE].

[130] LUX collaboration, D.S. Akerib et al., First results from the LUX dark matter experiment at the Sanford Underground Research Facility, Phys. Rev. Lett. 112 (2014) 091303 [arXiv:1310.8214] [INSPIRE].

[131] M. Drees and M. Nojiri, Neutralino-nucleon scattering revisited, Phys. Rev. D 48 (1993) 3483 [hep-ph/9307208] [INSPIRE].

[132] J. Hisano, K. Nakayama and M. Yamanaka, Implications of CDMS II result on Higgs sector in the MSSM, Phys. Lett. B 684 (2010) 246 [arXiv:0912.4701] [INSPIRE].

[133] A. Djouadi, M.M. Muhlleitner and M. Spira, Decays of supersymmetric particles: The program SUSY-HIT (SUspect-SdecaY-HDECAY-InTerface), Acta Phys. Polon. B 38 (2007) 635 [hep-ph/0609292] [INSPIRE]. 
[134] G. Bélanger, F. Boudjema, A. Pukhov and A. Semenov, MicrOMEGAs $s_{3}$ : A program for calculating dark matter observables, Comput. Phys. Commun. 185 (2014) 960 [arXiv: 1305.0237] [INSPIRE].

[135] A. Belyaev, N.D. Christensen and A. Pukhov, CalcHEP 3.4 for collider physics within and beyond the Standard Model, Comput. Phys. Commun. 184 (2013) 1729 [arXiv:1207.6082] [INSPIRE].

[136] D. Pierce and A. Papadopoulos, The complete radiative corrections to the gaugino and Higgsino masses in the minimal supersymmetric model, Nucl. Phys. B 430 (1994) 278 [hep-ph/9403240] [INSPIRE].

[137] M. Ibe, S. Matsumoto and R. Sato, Mass Splitting between Charged and Neutral Winos at Two-Loop Level, Phys. Lett. B 721 (2013) 252 [arXiv:1212.5989] [INSPIRE].

[138] XENON1T collaboration, E. Aprile, The XENON1T Dark Matter Search Experiment, Springer Proc. Phys. C12-02-22 (2013) 93 [arXiv: 1206.6288] [INSPIRE]. 